\title{
Asymmetric Standing Facilities: An Unexploited Monetary Policy Tool ${ }^{*}$
}

\author{
Gabriel Pérez Quirós ${ }^{\dagger}$ \\ Banco de España and CEPR \\ Hugo Rodríguez Mendizábal ${ }^{\ddagger}$ \\ Instituto de Análisis Económico (CSIC) and Barcelona GSE
}

November 26, 2009

\begin{abstract}
This paper analyzes the role of standing facilities in the determination of the demand for reserves in the overnight money market. In particular, we study how the asymmetric nature of the deposit and lending facilities could be used as a powerful policy tool for the simultaneous control of prices and quantities in the market for daily funds.
\end{abstract}

Keywords: Monetary policy implementation; standing facilities; overnight interest rates; fine-tuning operations.

JEL Codes: E52, E58, E43

\section{Introduction}

This paper analyzes the role of standing facilities in the determination of the demand for reserves in the overnight money market. In particular, we study how the asymmetric nature of the deposit and lending facilities could be used as a powerful policy tool for the simultaneous control of prices and quantities in the market for daily funds.

The motivation for such a study originates from the monetary policy decisions at the ECB and the Federal Reserve to respond to the current financial

\footnotetext{
*We would like to thank Juan Ayuso, Roberto Blanco, José Manuel Marqués, Arturo Mesón and participants at the internal seminar of the Banco de España for helpful suggestions. The views expressed in the paper are those of the authors and do not necessarily reflect the views of the Bank of Spain or the European System of Central Banks. Rodríguez Mendizábal acknowledges the support of the Barcelona GSE Research Network and of the Government of Catalonia.

†Servicio de Estudios, Banco de España, Alcala 50. 28014 Madrid. Spain. e-mail: gabriel.perez@bde.es.

¥Instituto de Análisis Económico. Campus UAB. 08193 Bellaterra Barcelona, Spain. email: hugo.rodriguez@iae.csic.es.
} 
crisis. Both the ECB and the Federal Reserve confronted the crisis by decreasing the rates of their refinancing operations but they felt obligated to complement this measure with changes in their operational procedures in the search of a new operational framework better suited for the new challenges associated with the financial turmoil . The ECB decided on two modifications to its monetary policy framework. First, on October 8th 2008, the corridor of standing facilities was reduced from 200 to 100 basis points centered around the interest rate of the main refinancing operations. Second, there were also adjustments in the tender procedure of these operations. Starting from the operation settled on October 15th 2008, the weekly main refinancing operations was carried out through a fixed rate tender procedure with full allotment. These two measures were designed to remain in place for as long as needed, and at least until the end of the first maintenance period of 2009, on January 20th. The ECB wished to communicate that it was going to steer liquidity towards balanced conditions in a way which was consistent with the objective to keep short term rates close to the interest rate on the main refinancing operation. ${ }^{1}$

Also on October 2008, the Federal Reserve made moves in a similar direction. First, on October 6th, the Fed announced the payment of interest on depository institutions' required and excess reserves balances. In particular, the interest rate for the excess reserves, which, in practice, is equivalent to a deposit rate, was fixed at 75 basis points below the target federal funds rate. The Board claimed that the payment of interest on excess reserves balances was going to give the Federal Reserve greater scope to use its lending programs to address conditions in credit markets while also maintaining the federal funds rate close to the target. Second, on October 22nd, the deposit rate was changed to 35 basis points below the target federal funds rate. Because since 2002 the discount window functions in practice as a lending facility, with the discount rate fixed at 50 basis points above the target for the federal funds rate, these decisions were intended to reduce the corridor for the overnight rate. In fact, as it was argued by the Board, a narrower spread between the target funds rate and the deposit rate would help foster trading in the funds market at rates closer to the target rate.

In the Euro area, most of the attention of the press and the first reactions of the markets participants came from the measure related to the full allotment. Having full allotment on fix-rate open market operations was seen as a direct measure to control market rates. On the other hand, because overnight rates will never be above the lending rate or below the deposit rate, the reduction of the spread between the lending and deposit rate was considered an obvious way to control interest rates. But this measure was mainly regarded as a purely technical point without much further implications on the behavior of market participants.

However, according to the ECB, the spread between the lending and deposit rates may have been relevant for the behavior of counterparties in the Eurosystem monetary policy operations. Apparently, agents reacted to the reduction in

\footnotetext{
${ }^{1}$ See the ECB press release of October 8th, 2008.
} 
this spread by asking in the main refinancing operations for much more reserves that they otherwise would have needed. This excess of reserves was then deposited in the deposit facility. To correct this situation, on January 15th 2009, the October 8th decision was revoked and the width of the corridor between the rates of the standing facilities was set to be 200 basis points again. ${ }^{2}$

In this paper we argue that, although this line of reasoning might not be wrong, the determination of the demand for excess reserves is somewhat more elaborated. First, we look at data and conclude that there is no clear cut relation between the demand for excess reserves and the amplitude of the corridor formed by the lending and deposit rates. The data shows how several factors that potentially could have an effect on the demand for reserves changed together with the width of the interest rate corridor. Second, we construct a model of reserve demand determination. We illustrate how the demand for funds depends on the position of the main refinancing rate within the band formed by the rates of the standing facilities and not on the width of that corridor. One conclusion of the model is that the position of the main refinancing rate with respect to the lending and deposit rates can be used as a monetary policy tool to control simultaneously interest rates and liquidity conditions. Furthermore, since March 2004 the ECB has been conducting fine-tuning operations at the end of the reserve maintenance periods to provide or drain any liquidity differential with respect to the ECB's estimated balanced reserve position. We use the model to understand the effect of this type of operations on the demand for excess reserves.

In previous work, we have demonstrated the important role standing facilities play in shaping the demand for reserves in "normal" times. Pérez Quirós and Rodríguez Mendizábal [16] and Gaspar, Pérez Quirós and Rodríguez Mendizábal [12] showed that the standing facilities have an important role in determining the distribution of overnight rates within the reserve maintenance period. The main assumption in these papers is a neutral monetary policy defined as the liquidity the system needs to fulfill reserve requirements. In this paper we adapt this previous framework to include the distress associated with a period of turmoil as the one experienced by financial markets after the summer of 2007 together with the existence of fine-tuning operations on the last day of the maintenance periods.

Obviously we are not the first ones in analyzing the role of the institutional framework for the dynamics of money markets. Starting from the seminal paper by Hamilton [13] where he analyzes possible reasons for the departure from the martingale hypothesis of the overnight rate in the US, different papers have studied the role of several elements of the monetary policy implementation framework. For example, Demiralp and Artuc [4] analyze the removal in 2003 of the stigma associated with the discount window in the US. For the Eurosystem, Ayuso and Repullo [1] and [2] analyze the optimal behavior of banks in the open market operations as a function of the type of auction design by the ECB and explain the overbidding behavior of banks in the first half of 2000. Valimaki

\footnotetext{
${ }^{2}$ See ECB [11], page 34 .
} 
[17] investigates the changes introduced in March 2004, namely, the timing of the reserve maintenance period to make them coincide with the meeting of the Governing Council and the reduction in the maturity of the main refinancing operations. He points out the importance of the size of the open market operations for the uncertainty of the outcome of the liquidity auction and links it to the increase in the price of the bids. His theoretical results are corroborated, among others, by the empirical applications of Linzert and Schmidt [15]. Finally, the implications of the recent financial crisis on the Euro money markets are analyzed in Cassola and Morana [3], Eisenschmidt, Hirsch and Linzert [6] as well as Eisenschmidt and Tapking [5]. In all these cases, the results are mainly empirical, evaluating the effect of the financial turmoil on the spread between the Eonia and the main refinancing operation rates or on the bidding behavior of the banks. These papers also try to show that the spreads between the secured and unsecured markets can not be explained just by higher credit risk.

As far as we know, not a single paper has formally studied the role of the standing facilities in the current crisis although Goodhart [14] has already pointed out the need for a careful analysis of the asymmetry in the interest rate corridor as a policy instrument. First he indicates that the parameters of the corridor "could become a flexible and subtle further instrument" of monetary policy so that "treating these parameters as a constant would be a waste of a good instrument". In fact, for the current crisis at hand he recommends that "before the recovery has properly begun, and while the financial system remains fragile $[. .$.$] the asymmetric bias should (penalize) reserve build up and$ (encourage) borrowing from the Fed".

The paper is structured as follows: Section 2 summarizes the recent developments in the Euro area money markets that motivate our work. Section 3 presents the theoretical model and its solution. Finally, section 4 concludes and discuss the policy implications of the results

\section{Recent developments in Euro-area money mar- kets}

In this section we provide some evidence to evaluate different explanations for the behavior of the demand for reserves in the Euro-area during the recent turmoil in financial markets. Figure 1 shows the daily use of the deposit facility as a percentage of total reserve requirements between January 3rd, 2007 and September 23rd, 2009. The figure also includes the two periods for which the width of the interest rate corridor formed by the lending and deposit rate has been below 200 basis points. The first period is represented by an area with vertical lines and goes from October 9th, 2008 until January 21st, 2009. In this period the interest rate corridor was 100 basis points wide. The second period started on May 13th, 2009 and runs until the end of the sample. This period is shown as an area with horizontal lines. In this case, the difference between the rates of the standing facilities was 150 basis points. These areas are shown in 
all the figures below. ${ }^{3}$

Between the beginning of the sample and September 25th, 2008, reserve balances at the deposit facility has averaged 0.26 percent of reserve requirements with a maximum of 5.8 percent. ${ }^{4}$ Then, by September 30th of that year this ratio increased to 48 percent of reserve requirements and stayed around 102 percent during the period between October 9th, 2008 and January 21st, 2009 when the spread between the lending and deposit rates was 100 basis points. Since mid-January 2009 this ratio has been decreasing reaching a minimum of 3.4 percent on June 24th, 2009. However, on June 25th, 2009, the use of the deposit facility started to rise again and reached its historical maximum of 145 percent of reserve requirements on July 3rd, 2009. ${ }^{5}$

Figure 2 presents the standing amounts of reserves injected through open market operations as a fraction of reserve requirements. Until the beginning of October 2008 this number fluctuated around 228 percent of reserve requirements. Then, between October 9th, 2008 and January 21st, 2009 it increased so that the average over this period was 364 percent. Since February 2009 this ratio has been decreasing until June 25th, 2009, when it raised again and reached 411 percent. This figure also includes the amount of autonomous factors as a fraction of reserve requirements. This series increased from a level of around 110 percent to just around 170 percent on October 2008 which means that changes in the behavior of autonomous factors cannot account for the larger recourse to the open market operations. Thus, these figures suggest that the increase in the use of the deposit facility has been financed through bigger amounts allotted at the open market operations of the ECB.

As pointed out in the Introduction, a natural explanation for the behavior of reserve demand has to do with changes in the cost of using the deposit facility. Because this cost is measured by the spread between the rate of the main refinancing operation and the deposit rate, in the two periods when this spread has decreased the appeal of the deposit facility has increased. This has resulted in larger reserve demands at the open market operations given the full allotment policy of the ECB after October 15th, 2009. Although this explanation might be correct, it may not capture all the factors behind the demand of reserves. A careful look at Figure 1 suggests that there ought to be other important elements to account for the behavior of reserve demand in the euro area. First, the increase in the use of the deposit facility does not exactly coincide with the reduction of the amplitude of the interest rate corridor. Banks started to move funds from their current accounts to the deposit facility in a significant manner

\footnotetext{
${ }^{3}$ In this paper we do not describe the institutional framework of the Euro area monetary policy. Interested readers that need some background in the Euro area monetary policy implementation rules should read European Central Bank [10]. For a description of the two major recent changes, the one in March 2004 and the one in October 2008, see ECB [7], ECB [8] and ECB [9].

${ }^{4}$ This value was reached on September 9 th, 2008 which was the end of a reserve maintenance period.

${ }^{5}$ On June 25th, 2009, the ECB started conducting liquidity-providing main refinancing operations with a year maturity. The larger use of the deposit facilities could be due to expectations of future rate increases.
} 
around two weeks before October 9th, 2008. Second, after the amplitude of the band went back to be 200 basis points on January 22nd, 2009, we witnessed a very gradual reduction in the use of the deposit facility, nothing comparable with the sudden peak observed on October 9th, 2008. Third, this fall in the recourse to the deposit facility occurred even when the cost of using this facility dropped again on May 13th, 2009. Finally, as suddenly as in October 2009, the deposit facility was again heavily used after June 25th, 2009, the day of the settlement of the first of the 1-year refinancing operations to provide liquidity, decided on the May 7th 2009 meeting of the Governing Council, although at this time the spread between the lending and deposit rate stayed constant at 150 basis points.

One possible element missing in the explanation above is the evolution of the risk involved in interbank lending as perceived by market participants. One way to think about the factors behind the demand of reserves is that market participants use the main refinancing operations and the deposit facility as a storage device in response to their perceptions of the risk involved in interbank lending. One commonly used measure of risk is the spread between the Euribor and the Eurepo at same maturities. The Euribor is the rate of uncollateralized loans in the interbank market while the Eurepo is the rate of the corresponding collateralized loan. Figure 3 shows the difference between the Euribor and the Eurepo at the 12-month maturity for the same sample period as in Figures 1 and 2. We can see how this spread started to rise at the end of August 2007 with a dramatic peak on October 2008. After moving around values close to 2 percentage points, the spread between the Euribor and Eurepo had a sharp decrease on December 5th, 2008 and it has been declining since then. Therefore, as the perception of market risk significantly increased on October, 2008, financial institutions responded by raising their demand for excess reserves. This was achieved by larger bids in the main refinancing operation. These larger amounts allotted by the ECB were then deposited at the deposit facility. Furthermore, the simultaneous narrowing of the interest rate corridor exacerbated the use of the demand for excess reserves and the use of the deposit facility as the cost of using this storage device decreased.

Again, although this explanation might be correct, a closer look at Figures 1 and 3 suggests that there still ought to be other important elements to account for the behavior of reserve demand in the euro area. First, perceived risk had already increased in a significant manner a year before market participants began using the deposit facility in sizable amounts. Furthermore, the conditions regarding the width of the interest rate corridor and the prize of risk around June and July of 2008 are basically the same as in March and April of 2009 but the use of the deposit facility is very different. Finally, between May 13th and July 7 th of 2009, the difference between the lending and deposit rates stayed constant and the prize of risk decreased. However, the use of the deposit facility increased substantially. ${ }^{6}$ From this evidence we conclude that neither the

\footnotetext{
${ }^{6}$ See footnote 5 for a possible explanation of the increase in the use of the deposit facility after June 25th, 2009.
} 
difference between the lending and deposit rates nor the market's perceptions on risk seem to provide satisfactory explanations of the behavior of the demand for excess reserves.

Another important element that might affect the demand for reserves might be related to the existence of fine-tuning operations on the last days of the reserve maintenance periods. The ECB has conducted a liquidity draining finetuning operation on the last day of each of the 11 reserve maintenance periods existent between November 2008 and September 2009. An important question is whether these fine tuning operations have drained all the excess liquidity in the system. To answer this question we use as a measure of the excess liquidity in the system the recourse to the deposit facility the previous to last day of the maintenance period. Figure 4 plots the amount of drainage, that is, the size of those 11 fine tuning operations, as well as the changes in the use of the deposit facility and in the aggregate current account of the system on the last day of the maintenance period. We normalize these quantities by our measure of excess liquidity so that all these numbers appear as fractions of the use of the deposit facility on the previous to last day of the maintenance period. For example, on the 7th of April of 2009, a fine tuning was done with a size of approximately 120 percent of the "excess liquidity" in the system, proxied with the use of the deposit facility on the 6th of April of 2009. Although the drainage of reserves was larger than the excess liquidity, this open market operation reduced the use of the deposit facility by just 60 percent. The other 60 percent implied a reduction of the current accounts of credit institutions in the ECB. ${ }^{7}$ We can see how the drainage of liquidity is always larger in absolute value than the reduction rate in the use of the deposit facility, and, sometimes, the drainage reaches values larger than 1 . This means that the drainage is larger than the outstanding balance of the deposit facility on the previous to last day of the maintenance period. However, the decrease rate in the deposit facility never reaches 1 so that there is always a significative fraction of use of the deposit facility on the last day. As Figure 4 shows, a significative part of the drainage of liquidity is obtained through reductions in current accounts. All these 11 operations were conducted through variable rate tenders. Therefore, even with fine tuning operations on the last day, there is an excess demand for reserves after the financial turmoil that can not be drained by the ECB.

Overnight market rates provide further evidence on the behavior of credit institutions. Figure 5 plots the spread between the Eonia and the rate of the main refinancing operations. We can see how this spread has been systematically negative after October 14th, 2008. This observation supports the idea that there is too much liquidity in the system.

Finally, for completeness, Figure 6 plots the use of the marginal lending facility over required reserves. To compare with Figure 1, it maintains the same range of values for the vertical axis. Unlike the use of the deposit facil-

\footnotetext{
${ }^{7}$ The variations in deposits and the variations in the current account do not always add to the size of the operation because variation in autonomous factors also contribute to changes in liquidity conditions. On those days, however, the contribution of autonomous factors is negligible.
} 
ity, recourse to the marginal lending facility has stayed in much smaller levels. The maximum value over the whole sample is just 13 percent with an average between October 9th, 2008 and January 21st, 2009 of 2.79 percent of reserve requirements.

In this paper we do not seek to provide an explanation of the observed behavior of the demand for excess reserves. Rather, we take as given the increase in demand for reserves associated with periods of turmoil in financial markets. We then construct a model in which, in such scenario, commercial banks have an incentive to demand sizable amounts of excess reserves at the main refinancing operations. Naturally, this state of affairs does not represent a desirable situation. On the one hand, funds injected by the central bank do not reach the money market and, therefore, cannot be channeled to those institutions who need them most. On the other hand, the central bank looses control on liquidity conditions as these funds can be put back in the market whenever financial institutions decide to do so.

We use the model to show how the demand for funds in the market depends on the relative position of the main refinancing rate with respect to the lending and deposit rates. In this sense, we illustrate how the demand for reserves is independent of the width of symmetric corridors around the main refinancing rate. One conclusion of the model is that the central bank can control the demand for reserves and the use of the deposit facility by carefully choosing the position of the main refinancing rate within the corridor defined by the rates of the standing facilities. Furthermore, keeping constant the amplitude of this corridor ensures the same degree of control of market rates. Thus, the width together with the degree of asymmetry of the interest rate band can be used as two monetary policy tools to control simultaneously interest rates and liquidity conditions in the interbank market. Finally, as the fine-tuning operation at the end of the maintenance period affects the expected profits from reserve management perceived by market participants, we also show the role that such operations have in shaping the demand for excess reserves.

\section{The theoretical model}

\subsection{The setup}

This section presents a dynamic general equilibrium model of the Eurosystem overnight money market. The main features of the model are based on Pérez Quirós and Rodríguez Mendizábal [16]. Assume an economy inhabited by a central bank and a continuum of commercial banks with measure one. These commercial banks maintain deposits, called current accounts or reserves, with the central bank in order to fulfil reserve requirements and payments responsibilities. Every day an interbank market opens where banks can exchange reserves through loans with an overnight maturity.

When deciding how much funds to loan or to borrow from the market, banks consider the constraints associated with the operational framework of the cen- 
tral bank. This framework is composed of three elements. The first one refers to certain restrictions on the current accounts hold by commercial banks. Reserve balances of commercial banks cannot be negative by the end of each day and the accumulated balance over each reserve maintenance period has to be large enough to meet required reserves, that is, it cannot be smaller than a number $R>0$. This number $R$ corresponds to the level of required reserves and is pre-determined. The reserve maintenance period has a length of $T=2$ days. The second element consists of two standing facilities provided by the central bank. There is a lending facility where banks can borrow funds at the interest rate $i^{l}$ and a deposit facility where banks can deposit funds at the rate $i^{d}$. These facilities are always available to commercial banks. The third element includes the central bank's open market operations (OMOs). We assume there is a main refinancing operation performed at the beginning of the reserve maintenance period. This OMO has a maturity of $T=2$ days. There also exists the possibility of an overnight fine-tuning operation at the very end of the maintenance period. From the individual point of view of commercial banks this fine-tuning operation occurs with probability $p^{f}$. If performed, the fine-tuning operation may be liquidity absorbing or providing depending on the aggregate liquidity conditions. Both OMOs allot bids fully and are conducted as fixed rate tenders at the rate $i^{o}$, with $i^{d}<i^{o}<i^{l}$.

The theoretical design of the fine-tuning operation is novel and deserves further explanation. We wanted to model two features of this operation. First, the ECB does not always perform these operations so that from the point of an individual bank there is uncertainty about the possibility to accommodate individual reserve imbalances at the end of the maintenance period. On the other hand, these operations, when performed are usually done through variable rate tenders. ${ }^{8}$ This means the rate at which the fine-tuning operation is settled should be between the rate of the main refinancing operation and the rate of the corresponding standing facility depending on whether the operation is liquidity draining or providing. As will be seen below, the subjective probability $p^{f}$ can be interpreted to capture this feature of the operational framework of the Eurosystem.

The problem for each bank $j \in[0,1]$ is as follows. Because each bank starts every maintenance period without funds, and because the OMO has a maturity of $T$ days, each maintenance period is an independent entity so we can concentrate on the decisions made by banks on an isolated maintenance period. As they start day 1 with no reserves, at the beginning of that day each bank has to decide how much reserves, $a_{1}^{j}$, to get from the OMO of the central bank. Once this decision is taken, a perfectly competitive overnight interbank market opens every day where banks decide how much funds to loan out or borrow at an interest rate $i_{t}$. Banks make these decisions in order to maximize their expected sum of profits from reserve management over all $T$ days of the maintenance period. Apart from aggregate variables, the information

\footnotetext{
${ }^{8}$ As explained in section 2 , this has been the case for all the fine-tuning operations performed at the end of the reserve maintenance periods between November 2008 and September 2009 .
} 
needed by an individual bank $j$ to make these decisions is summarized by its reserve position $s_{t}^{j}=\left(a_{t}^{j}, r_{t}^{j}\right)$. This position is defined by its current account at the beginning of each day, $a_{t}^{j}$, and the amount of reserves bank $j$ has to accumulate from day $t$ until day $T$ to satisfy its reserve requirement, also know as its deficiency, $r_{t}^{j}$. Let $m_{t}^{j}$ and $b_{t}^{j}$ be the amount of funds kept by a bank and the ones loaned out in the market at day $t$, respectively. These magnitudes have to satisfy

$$
m_{t}^{j}+b_{t}^{j}=a_{t}^{j}
$$

and

$$
m_{t}^{j} \geq 0 \text {. }
$$

After the market closes, bank $j$ receives a liquidity shock, $\varepsilon_{t}^{j}$. If $\varepsilon_{t}^{j}$ is positive, the balance of the bank increases and if it is negative the balance decreases. We assume these shocks are i.i.d. across time and banks and are distributed according to a distribution $G$ with mean 0 and standard deviation $\sigma$. At the same time, we want to model the subjective perceptions by individual banks about the possibility of facing tough liquidity conditions in the course of the current reserve maintenance period. This ex-ante subjective perception of aggregate liquidity conditions can be summarized by the possibility of a permanent aggregate liquidity drain equal to $\mu=e<0$. The subjective probability assigned to this event is $p^{\mu}$. On the other hand, the reserve maintenance period may be characterized by normal payment responsibilities. In this case there is no liquidity drain so that $\mu=0$. Banks assign a probability $1-p^{\mu}$ to this event. This aggregate uncertainty is realized on day 1 after the OMO has taken place and before the interbank market opens.

We assume that interests on interbank lending are paid separately so they are not capitalized. ${ }^{9}$ This assumption means that current accounts at the beginning of day 2 evolve exogenously according to ${ }^{10}$

$$
a_{2}^{j}=a_{1}^{j}+\varepsilon_{1}^{j}+\mu .
$$

On the other hand, reserve deficiencies on day $2, r_{2}^{j}$, that is, the level of the current account of bank $j$ at the end of day 2 needed to satisfy its reserve requirement, evolve as

$$
r_{2}^{j}=\max \left\{0, R-\max \left[0, m_{1}^{j}+\varepsilon_{1}^{j}\right]\right\} .
$$

That is, the reserve balance of the bank at the end of day $1\left(m_{1}^{j}+\varepsilon_{1}^{j}\right)$ is accumulated only if it is positive and deficiencies cannot be negative. Thus, what matters is fulfilling the requirement of accumulating $R$ reserves over the $T=2$ days and not how much reserves are accumulated on each particular

\footnotetext{
${ }^{9}$ Interest rates from exchanging reserves in the interbank market are usually paid at the end of the maintenance period. Thus, they are part of the profits of the banks but should not affect the daily evolution of the beginning-of-day reserves.

${ }^{10}$ The reader should remember that trading in the market is done with an overnight maturity. This means that the intertemporal evolution of reserves for an individual bank does not depend on the decision made about $m_{t}^{j}$ or $b_{t}^{j}$ but only on the realization of the shock $\epsilon_{t}^{j}$.
} 
day. Notice, deficiencies are nonincreasing over time. Furthermore, once the deficiency is zero, additional reserves are accumulated in excess. We refer to this situation by saying that a bank is "locked-in". In other words, zero is an absorbing state for deficiencies.

\subsection{The problem of bank $j$ at $T=2$}

The model is solved by backward induction. With this method, we first solve the problem for all possible individual and aggregate states and dates starting at day $T=2$ and working backwards towards the beginning of the maintenance period. This information allows us to find bids in the fine-tuning operation as well as the demand or supply of funds in the interbank market for every bank on every day and to find the sequence of equilibrium rates conditioned on the realization of the aggregate state. Once we know the market clearing rates we are able to compute the profits from having reserves through the maintenance period. Notice these sequences of profits and equilibrium rates are conditioned on the initial level of reserves for each bank, $a_{1}^{j}, j \in[0,1]$, that were obtained at the open market operation at the beginning of day 1. Then it is possible to see how many reserves banks are willing to obtain from the central bank by comparing the OMO rate with the expected value of those funds throughout the maintenance period.

\subsubsection{The fine-tuning operation}

During $T=2$, bank $j$ decides to leave $m_{2}^{j}$ in the current account at the central bank. Then, it is hit by the liquidity shock $\varepsilon_{2}^{j}$. This means that by the time the fine-tuning operation occurs, bank $j$ has a current account balance of $m_{2}^{j}+\varepsilon_{2}^{j}$ and a deficiency of $r_{2}^{j}$. With this information, bank $j$ knows whether it will satisfy the reserve requirement and will have excess reserves or not. Let $f_{2}^{j}$ be the bid at the fine-tuning operation. The end-of-maintenance period costs of reserve management, $c_{2}^{j}\left(m_{2}^{j}+\varepsilon_{2}^{j}, r_{2}^{j}, f_{2}^{j}\right)$, are

$$
\begin{aligned}
c_{2}^{j}\left(m_{2}^{j}+\varepsilon_{2}^{j}, r_{2}^{j}, f_{2}^{j}\right)= & i^{o} f_{2}^{j}+i^{l}\left(r_{2}^{j}-m_{2}^{j}-\varepsilon_{2}^{j}-f_{2}^{j}\right) I\left[r_{2}^{j}>m_{2}^{j}+\varepsilon_{2}^{j}+f_{2}^{j}\right] \\
& +i^{d}\left(r_{2}^{j}-m_{2}^{j}-\varepsilon_{2}^{j}-f_{2}^{j}\right) I\left[r_{2}^{j} \leq m_{2}^{j}+\varepsilon_{2}^{j}+f_{2}^{j}\right],
\end{aligned}
$$

with $I[x]$ being an indicator function that takes value 1 when the statement in brackets is true. The first term on the right hand side represents the costs of borrowing from the central bank at the interest rate $i^{o}$. If the central bank conducts a liquidity draining fine-tuning OMO $f_{2}^{j}$ would be negative and that term would represent a revenue for bank $j$. The second term is the costs of using the marginal lending facility at the rate $i^{l}$. This happens when accumulated reserves are not enough to satisfy the reserve requirement $\left(r_{2}^{j}>m_{2}^{j}+\varepsilon_{2}^{j}+f_{2}^{j}\right)$. The third term is the revenues from recourse to the deposit facility at the rate $i^{d}$. This happens when the bank has excess reserves at the end of the day $\left(r_{2}^{j} \leq m_{2}^{j}+\varepsilon_{2}^{j}+f_{2}^{j}\right)$. Given the rates of the standing facilities $\left(i^{d}\right.$ and $\left.i^{l}\right)$ and 
the one of the fine-tuning operation $\left(i^{d}<i^{o}<i^{l}\right)$, bank $j$ will always prefer to use the OMO rather than the facilities to balance its current account.

The only constraint bank $j$ faces is that the sign of the fine-tuning operation should agree with its liquidity needs. The central bank decides whether the finetuning operation drains or provides liquidity attending at aggregate liquidity conditions by comparing aggregate current accounts, $A_{2},{ }^{11}$

$$
A_{2}=\int a_{2}^{j} d j
$$

with aggregate deficiencies, $R_{2}$,

$$
R_{2}=\int r_{2}^{j} d j
$$

Thus, if there is an aggregate shortage of liquidity, i.e. if $A_{2}-R_{2}<0$, the finetuning operation will be liquidity providing and the bid of bank $j$ is constrained to be positive, i.e. $f_{2}^{j} \geq 0$. On the contrary, if there is an aggregate excess of liquidity, i.e. if $A_{2}-R_{2}>0$, the fine-tuning operation will be liquidity draining and the bid of bank $j$ is constrained to be negative, i.e. $f_{2}^{j} \leq 0$. Finally in the case of balanced liquidity conditions, i.e. if $A_{2}-R_{2}=0$, there will be no fine-tuning operation and the bid of bank $j$ will have to be zero, i.e. $f_{2}^{j}=0$. It is important to notice that aggregate liquidity conditions are endogenous as they depend on the bids at the initial main refinancing operation.

The problem of bank $j$ in the fine tuning operation is to decide the bid to minimize costs of reserve management. This problem can be written as

$$
V_{2}^{f}\left(m_{2}^{j}+\varepsilon_{2}^{j}, r_{2}^{j} ; A_{2}-R_{2}\right)=\min _{f_{2}^{j}} c_{2}^{j}\left(m_{2}^{j}+\varepsilon_{2}^{j}, r_{2}^{j}, f_{2}^{j}\right)
$$

subject to

$$
\begin{array}{ll}
f_{2}^{j} \geq 0 & \text { if } A_{2}-R_{2}<0 \\
f_{2}^{j}=0 & \text { if } A_{2}-R_{2}=0 \\
f_{2}^{j} \leq 0 & \text { if } A_{2}-R_{2}>0 .
\end{array}
$$

Given the linearity of the objective function the optimal decision will be at a corner, either $f_{2}^{j}=0$ or $f_{2}^{j}=r_{2}^{j}-m_{2}^{j}-\varepsilon_{2}^{j}$, depending on whether the reserve position of bank $j$ coincides or not with the aggregate reserve position of the market as a whole. Thus, if there is an aggregate reserve deficiency, $A_{2}-R_{2}<0$, so that the fine-tuning operation is liquidity providing, bank $j$ will bid zero, $f_{2}^{j}=0$, if it has excess reserves $\left(r_{2}^{j} \leq m_{2}^{j}+\varepsilon_{2}^{j}\right)$ or all its reserve shortage, $f_{2}^{j}=r_{2}^{j}-m_{2}^{j}-\varepsilon_{2}^{j}$, if itself does not have enough reserves to satisfy its requirement $\left(r_{2}^{j}>m_{2}^{j}+\varepsilon_{2}^{j}\right)$. In that case, the minimized costs are

$$
\begin{aligned}
V_{2}^{f}\left(m_{2}^{j}+\varepsilon_{2}^{j}, r_{2}^{j} ; A_{2}-R_{2}<\right. & 0)=i^{o}\left(r_{2}^{j}-m_{2}^{j}-\varepsilon_{2}^{j}\right) I\left[r_{2}^{j}>m_{2}^{j}+\varepsilon_{2}^{j}\right] \\
& +i^{d}\left(r_{2}^{j}-m_{2}^{j}-\varepsilon_{2}^{j}\right) I\left[r_{2}^{j} \leq m_{2}^{j}+\varepsilon_{2}^{j}\right],
\end{aligned}
$$

\footnotetext{
${ }^{11}$ In what follows, capital letters refer to aggregate variables.
} 
and the partial derivative with respect to the current account holdings of the bank is

$$
\frac{\partial V_{2}^{f}\left(m_{2}^{j}+\varepsilon_{2}^{j}, r_{2}^{j} ; A_{2}-R_{2}<0\right)}{\partial m_{2}^{j}}=-i^{o} I\left[r_{2}^{j}>m_{2}^{j}+\varepsilon_{2}^{j}\right]-i^{d} I\left[r_{2}^{j} \leq m_{2}^{j}+\varepsilon_{2}^{j}\right]
$$

On the other hand, if there are aggregate excess reserves, $A_{2}-R_{2}>0$, so that the fine-tuning operation is liquidity draining, bank $j$ will bid all its excess reserves, $f_{2}^{j}=r_{2}^{j}-m_{2}^{j}-\varepsilon_{2}^{j}$, if it has reserves over its requirement $\left(r_{2}^{j} \leq m_{2}^{j}+\varepsilon_{2}^{j}\right)$ or zero, $f_{2}^{j}=0$, if itself does not have enough reserves to satisfy its requirement $\left(r_{2}^{j}>m_{2}^{j}+\varepsilon_{2}^{j}\right)$. In that case, the minimized costs are

$$
\begin{aligned}
V_{2}^{f}\left(m_{2}^{j}+\varepsilon_{2}^{j}, r_{2}^{j} ; A_{2}-R_{2}>\right. & 0)=i^{l}\left(r_{2}^{j}-m_{2}^{j}-\varepsilon_{2}^{j}\right) I\left[r_{2}^{j}>m_{2}^{j}+\varepsilon_{2}^{j}\right] \\
& +i^{o}\left(r_{2}^{j}-m_{2}^{j}-\varepsilon_{2}^{j}\right) I\left[r_{2}^{j} \leq m_{2}^{j}+\varepsilon_{2}^{j}\right]
\end{aligned}
$$

and the partial derivative with respect to the current account holdings of the bank is

$$
\frac{\partial V_{2}^{f}\left(m_{2}^{j}+\varepsilon_{2}^{j}, r_{2}^{j} ; A_{2}-R_{2}>0\right)}{\partial m_{2}^{j}}=-i^{l} I\left[r_{2}^{j}>m_{2}^{j}+\varepsilon_{2}^{j}\right]-i^{o} I\left[r_{2}^{j} \leq m_{2}^{j}+\varepsilon_{2}^{j}\right] .
$$

Finally, with balanced liquidity conditions, $A_{2}-R_{2}>0$, there is no fine-tuning operation, $f_{2}^{j}=0$. In that case, the minimized costs are

$$
\begin{aligned}
V_{2}^{f}\left(m_{2}^{j}+\varepsilon_{2}^{j}, r_{2}^{j} ; A_{2}-R_{2}=\right. & 0)=i^{l}\left(r_{2}^{j}-m_{2}^{j}-\varepsilon_{2}^{j}\right) I\left[r_{2}^{j}>m_{2}^{j}+\varepsilon_{2}^{j}\right] \\
& +i^{d}\left(r_{2}^{j}-m_{2}^{j}-\varepsilon_{2}^{j}\right) I\left[r_{2}^{j} \leq m_{2}^{j}+\varepsilon_{2}^{j}\right]
\end{aligned}
$$

and the partial derivative with respect to the current account holdings of the bank is

$$
\frac{\partial V_{2}^{f}\left(m_{2}^{j}+\varepsilon_{2}^{j}, r_{2}^{j} ; A_{2}-R_{2}=0\right)}{\partial m_{2}^{j}}=-i^{l} I\left[r_{2}^{j}>m_{2}^{j}+\varepsilon_{2}^{j}\right]-i^{d} I\left[r_{2}^{j} \leq m_{2}^{j}+\varepsilon_{2}^{j}\right] .
$$

Notice what the fine-tuning operation does. It changes the marginal value of funds at the end of the reserve maintenance period when the ECB computes reserve requirements. In this sense, if the individual bank reserve position coincides with the aggregate one, the existence of the fine-tuning operation deviates the opportunity cost of funds from the corresponding standing facility $\left(i^{l}\right.$ or $i^{d}$ depending on whether the operation is liquidity providing or draining, respectively) to the one of the open market operation $\left(i^{o}\right)$.

\subsubsection{The interbank market at $t=2$}

Because all banks start identical, they will demand the same amount of reserves at the initial OMO $\left(a_{1}^{j}=A_{1}\right.$, for all $\left.j \in[0,1]\right)$ and there will be no borrowing 
or lending at the first day of the maintenance period $\left(b_{1}^{j}=0\right.$, for all $\left.j \in[0,1]\right)$. This means that the distribution of individual states $\left(a_{2}^{j}, r_{2}^{j}\right.$ for all $\left.j \in[0,1]\right)$ is exogenous at $t=2$ and will only depend on the particular distribution of individual shocks, which includes the realization of the aggregate liquidity drain $\mu$, either $e<0$ with probability $p^{\mu}$ or 0 with probability $1-p^{\mu}$. The distribution of initial reserves at $t=2$ is given by

$$
a_{2}^{j}=A_{1}+\mu+\varepsilon_{1}^{j},
$$

and is distributed according to the distribution $G$ but with mean $A_{1}+\mu$ and standard deviation $\sigma$. The determination of individual deficiencies is as follows:

$$
r_{2}^{j}= \begin{cases}R, & \text { for banks for which } \varepsilon_{1}^{j} \leq-A_{1}-\mu, \\ R-A_{1}-\varepsilon_{1}^{j}, & \text { for banks for which }-A_{1}-\mu<\varepsilon_{1}^{j} \leq R-A_{1}-\mu, \\ 0, & \text { for banks for which } R-A_{1}-\mu<\varepsilon_{1}^{j} .\end{cases}
$$

This means that the distribution of individual deficiencies at $T=2$, is described by a truncated distribution. The truncation points are $r_{2}^{j}=R$ and $r_{2}^{j}=0$ with mass equal to

$$
\operatorname{prob}\left(r_{2}^{j}=R\right)=G\left(-A_{1}-\mu\right),
$$

and

$$
\operatorname{prob}\left(r_{2}^{j}=0\right)=1-G\left(R-A_{1}-\mu\right),
$$

respectively, where $G(x)$ represents the distribution of liquidity shocks. Notice the joint distribution of individual states depends on the initial amount of reserves allotted at the main refinancing OMO, $A_{1}$.

Let $\pi_{2}^{j}\left(a_{2}^{j}, r_{2}^{j} ; \mu\right)$ be the profits of bank $j$ at day $T=2$. Given the realization of the aggregate liquidity drain, $\mu$, and the individual state of the bank, $s_{2}^{j}=$ $\left(a_{2}^{j}, r_{2}^{j}\right)$, the decision problem at the interbank market can be written as follows. Bank $j$ will decide on the supply of funds in the market, $b_{2}^{j}=a_{2}^{j}-m_{2}^{j}$, to maximize expected profits

$$
\max _{b_{2}^{j}} E_{2}\left[\pi_{2}^{j}\left(s_{2}^{j} ; \mu\right)\right] .
$$

In this expression, profits are equal to

$$
\begin{aligned}
\pi_{2}^{j}\left(s_{2}^{j} ; \mu\right)= & i_{2}(\mu) b_{2}^{j}-p^{f} V_{2}^{f}\left(m_{2}^{j}+\varepsilon_{2}^{j}, r_{2}^{j} ; A_{2}-R_{2}\right) \\
& -\left(1-p^{f}\right) V_{2}^{f}\left(m_{2}^{j}+\varepsilon_{2}^{j}, r_{2}^{j} ; A_{2}-R_{2}=0\right) .
\end{aligned}
$$

The first term on the right hand side represents the revenues (costs) of lending (borrowing) in the market at the interest rate $i_{2}(\mu)$. In that term we wanted to make explicit the idea that the equilibrium rate depends on the realization of the aggregate liquidity shock, $\mu$. The second term and third term represent the end-of-maintenance period costs of liquidity management. With probability $p^{f}$ there will be a fine-tuning operation whose cost depends on the current account holding of the bank, $m_{2}^{j}+\varepsilon_{2}^{j}$, and its deficiency, $r_{2}^{j}$, as well as on the aggregate 
liquidity conditions, $A_{2}-R_{2}$. With probability $1-p^{f}$ there will be no fine-tuning operation. From an individual point of view this event is captured by the costs in case aggregate liquidity conditions are balanced, $V_{2}^{f}\left(m_{2}^{j}+\varepsilon_{2}^{j}, r_{2}^{j} ; A_{2}-R_{2}=0\right)$.

As the particular solution depends on the aggregate liquidity conditions, we can solve the problem for the three cases considered so far. In case of an aggregate liquidity shortage, $A_{2}-R_{2}<0$, substituting (1) and (6) in (8) yields expected profits equal to

$$
\begin{aligned}
E_{2}\left[\pi_{2}^{j}\left(s_{2}^{j} ; \mu\right)\right]= & i_{2}(\mu) b_{2}^{j}-i^{d}\left(r_{2}^{j}+b_{2}^{j}-a_{2}^{j}\right)\left[1-G\left(r_{2}^{j}+b_{2}^{j}-a_{2}^{j}\right)\right] \\
& -\left[p^{f} i^{o}+\left(1-p^{f}\right) i^{l}\right]\left(r_{2}^{j}+b_{2}^{j}-a_{2}^{j}\right) G\left(r_{2}^{j}+b_{2}^{j}-a_{2}^{j}\right) \\
& +\left[p^{f} i^{o}+\left(1-p^{f}\right) i^{l}\right] \int_{-\infty}^{r_{2}^{j}+b_{2}^{j}-a_{2}^{j}} \varepsilon g(\varepsilon) d \varepsilon \\
& +i^{d} \int_{r_{2}^{j}+b_{2}^{j}-a_{2}^{j}}^{\infty} \varepsilon g(\varepsilon) d \varepsilon,
\end{aligned}
$$

where $g(x)$ is the density function of the liquidity shock. The first order condition for a maximum is

$$
i_{2}(\mu)=i^{d}+\left[p^{f} i^{o}+\left(1-p^{f}\right) i^{l}-i^{d}\right] G\left(r_{2}^{j}+b_{2}^{j}-a_{2}^{j}\right) .
$$

This expression determines the supply of funds by bank $j$ on the last day of the reserve maintenance period, $b_{2}^{j}$, as a function of its state, summarized by its current account balance at the beginning of the day, $a_{2}^{j}$, and its reserve deficiency, $r_{2}^{j}{ }^{12}$ The interpretation is simple. Banks will supply funds in the market up to the point where the marginal value of an extra unit of liquidity equates its expected opportunity cost. The marginal return is represented by the market rate, $i_{2}(\mu)$. The marginal opportunity cost depends on the situation the bank ends up at the end of the day. In this sense, two events can happen. On the one hand, the bank may satisfy the reserve requirement and end up with excess reserves. This event happens with probability $1-G\left(r_{2}^{j}+b_{2}^{j}-a_{2}^{j}\right)$. In such a case, the bank will deposit the excess reserves at the deposit facility which implies a low opportunity cost equal to the deposit rate $i^{d}$. On the other hand, the bank may not satisfy the reserve requirement and will have to obtain the necessary funds from the central bank. This event happens with probability $G\left(r_{2}^{j}+b_{2}^{j}-a_{2}^{j}\right)$. In such a case the opportunity cost will depend on whether there is a fine-tuning operation or not. With probability $p^{f}$ there is a liquidity providing fine-tuning operation and the cost of funds would be $i^{o}{ }^{13} \mathrm{With}$

\footnotetext{
${ }^{12}$ This expression assumes an interior solution. If $\widehat{b}_{2}^{j}$ represents the solution of that expression, the decision on supply of funds have to satisfy $b_{2}^{j}=\min \left[\widehat{b}_{2}^{j}, \max \left(0, a_{2}^{j}\right)\right]$.

${ }^{13}$ Remember we are conditioning on the event of an aggregate liquidity shortage so that the fine-tuning operation is liquidity providing.
} 
probability $1-p^{f}$ there is no fine-tuning operation and the bank will need to go to the lending facility to obtain funds at the cost $i^{l}$. Here we can see the role of the probability $p^{f}$ in interpreting the fine-tuning operation in the model as performed through a variable rate tender. The ex-ante marginal cost of funds in case the bank finds itself with a liquidity shortage will be between $i^{o}$ and $i^{l}$ as it is the case with the variable rate operations.

From (9) it is easy to see that the marginal value of an additional unit of reserves is

$$
\frac{\partial V_{2}\left(s_{2}^{j} ; \mu\right)}{\partial a_{2}^{j}}=i^{d}+\left[p^{f} i^{o}+\left(1-p^{f}\right) i^{l}-i^{d}\right] G\left(r_{2}^{j}+b_{2}^{j}-a_{2}^{j}\right) .
$$

At the same time, the marginal value of an additional unit of deficiency is

$$
\frac{\partial V_{2}\left(s_{2}^{j} ; \mu\right)}{\partial r_{2}^{j}}=-\frac{\partial V_{2}\left(s_{2}^{j} ; \mu\right)}{\partial a_{2}^{j}} .
$$

The intuition of this expression is easy. Starting the last day with an extra unit of deficiency means not being able to loan a unit of reserves in the market. The marginal cost of this change is the marginal value of a unit of reserves. Expressions (11) and (12) will be used below.

The other cases are handled similarly and have analogous intuitions. In case of aggregate excess liquidity, $A_{2}-R_{2}<0$, substituting (1) and (6) in (8) and taking first order conditions yields

$$
i_{2}(\mu)=\left[p^{f} i^{o}+\left(1-p^{f}\right) i^{d}\right]+\left[i^{l}-p^{f} i^{o}-\left(1-p^{f}\right) i^{d}\right] G\left(r_{2}^{j}+b_{2}^{j}-a_{2}^{j}\right),
$$

with

$$
\frac{\partial V_{2}\left(s_{2}^{j} ; \mu\right)}{\partial a_{2}^{j}}=i^{d}+\left[p^{f} i^{o}+\left(1-p^{f}\right) i^{l}-i^{d}\right] G\left(r_{2}^{j}+b_{2}^{j}-a_{2}^{j}\right)
$$

and

$$
\frac{\partial V_{2}\left(s_{2}^{j} ; \mu\right)}{\partial r_{2}^{j}}=-\frac{\partial V_{2}\left(s_{2}^{j} ; \mu\right)}{\partial a_{2}^{j}}
$$

Finally, when there are balanced liquidity conditions, $A_{2}-R_{2}<0$, substituting (1) and (6) in (8) and taking first order conditions yields

$$
i_{2}(\mu)=i^{d}+\left(i^{l}-i^{d}\right) G\left(r_{2}^{j}+b_{2}^{j}-a_{2}^{j}\right)
$$

with

$$
\frac{\partial V_{2}\left(s_{2}^{j} ; \mu\right)}{\partial a_{2}^{j}}=i^{d}+\left(i^{l}-i^{d}\right) G\left(r_{2}^{j}+b_{2}^{j}-a_{2}^{j}\right)
$$

and

$$
\frac{\partial V_{2}\left(s_{2}^{j} ; \mu\right)}{\partial r_{2}^{j}}=-\frac{\partial V_{2}\left(s_{2}^{j} ; \mu\right)}{\partial a_{2}^{j}}
$$




\subsubsection{The interbank market at $t=1$}

At $t=1$ all banks start identical with $a_{1}^{j}=A_{1}$ and $r_{1}^{j}=R$. Define the value function $V_{1}\left(s_{1}^{j} ; \mu\right)$ as the maximized expected profits from days 1 and 2 ,

$$
V_{1}\left(s_{1}^{j} ; \mu\right)=\max _{b_{1}^{j}} E_{1}\left[\pi_{1}^{j}\left(s_{1}^{j} ; \mu\right)+V_{2}\left(s_{2}^{j} ; \mu\right)\right] .
$$

Profits at day 1 are equal to

$$
\begin{aligned}
\pi_{1}^{j}\left(s_{t}^{j} ; \mu\right)= & i_{1}(\mu) b_{1}^{j}+i^{l}\left(m_{1}^{j}+\varepsilon_{1}^{j}\right) I\left[m_{1}^{j}+\varepsilon_{1}^{j}<0\right] \\
& -i^{d}\left(r_{1}^{j}-m_{1}^{j}-\varepsilon_{1}^{j}\right) I\left[m_{1}^{j}+\varepsilon_{1}^{j}>r_{1}^{j}\right] .
\end{aligned}
$$

Notice the bank goes to the lending facility if it has an overdraft $\left(m_{1}^{j}+\varepsilon_{1}^{j}<0\right)$ and not if it does not satisfy the reserve requirement as on day $T=2$. Also notice that, although banks need to know the day of the period, this variable enters the problem by changing the form of the value function which links the state to the objective of the bank. This is why it is not included as a state variable but indexing the function $V$.

As before, using (1) and taking expectations produces

$$
\begin{aligned}
E_{1}\left[\pi_{1}^{j}\left(s_{1}^{j} ; \mu\right)\right] & =i_{1}(\mu) b_{1}^{j}-i^{l}\left(b_{1}^{j}-A_{1}-\mu\right) G\left(b_{1}^{j}-A_{1}-\mu\right) \\
& -i^{d}\left(R+b_{1}^{j}-A_{1}-\mu\right)\left[1-G\left(R+b_{1}^{j}-A_{1}-\mu\right)\right] \\
& +i^{l} \int_{-\infty}^{b_{1}^{j}-A_{1}-\mu} \varepsilon g(\varepsilon) d \varepsilon+i^{d} \int_{R+b_{1}^{j}-A_{1}-\mu}^{\infty} \varepsilon g(\varepsilon) d \varepsilon .
\end{aligned}
$$

To compute the second term in (19) we make use of the distribution of $r_{2}^{j}$. Then

$$
\begin{aligned}
E_{1}\left[V_{2}\left(s_{2}^{j} ; \mu\right)\right]= & \int_{-\infty}^{b_{1}^{j}-A_{1}-\mu} V_{2}\left(R, a_{2}^{j} ; \mu\right) g(\varepsilon) d \varepsilon \\
& +\int_{b_{1}^{j}-A_{1}-\mu}^{R+b_{1}^{j}-A_{1}-\mu} V_{2}\left(R+b_{1}^{j}-A_{1}-\mu-\varepsilon, a_{2}^{j} ; \mu\right) g(\varepsilon) d \varepsilon \\
& +\int_{R-b_{1}^{j}-A_{1}-\mu}^{\infty} V_{2}\left(0, a_{2}^{j} ; \mu\right) g(\varepsilon) d \varepsilon .
\end{aligned}
$$

The first order condition with respect to $b_{1}^{j}$ evaluated at $b_{1}^{j}=0$ gives

$$
\begin{gathered}
i_{1}(\mu)=i^{l} G\left(-A_{1}-\mu\right)+i^{d}\left[1-G\left(R-A_{1}-\mu\right)\right] \\
\quad-\int_{-A_{1}-\mu}^{R-A_{1}-\mu} \frac{\partial V_{2}\left(r_{2}^{j}, a_{2}^{j} ; \mu\right)}{\partial r_{2}^{j}} g(\varepsilon) d \varepsilon .
\end{gathered}
$$


Assuming an interior solution this expression becomes

$$
\begin{aligned}
i_{1}(\mu)= & i^{l} G\left(-A_{1}-\mu\right)+i^{d}\left[1-G\left(R-A_{1}-\mu\right)\right] \\
& +i_{2}(\mu)\left[G\left(R-A_{1}-\mu\right)-G\left(-A_{1}-\mu\right)\right] .
\end{aligned}
$$

The partial derivative of the value function with respect to $a_{1}^{j}$ is

$$
\frac{\partial V_{1}\left(s_{1}^{j} ; \mu\right)}{\partial a_{1}^{j}}=i_{1}(\mu)+\int_{-\infty}^{\infty} \frac{\partial V_{2}\left(r_{2}^{j}, a_{2}^{j} ; \mu\right)}{\partial a_{2}^{j}} g(\varepsilon) d \varepsilon=i_{1}(\mu)+i_{2}(\mu) .
$$

What is the value that a bank associates with having an additional unit of reserves at day 1? This bank can lend that unit on day 1 and on day 2. This decision will not affect its state on any day and will increase its profits by $i_{1}(\mu)+i_{2}(\mu)$. In other words, having an additional unit of reserves on day 1 means, on average, a permanent addition for the balance of the bank and, therefore, its value is the accumulated expected revenue of that unit for the rest of the reserve maintenance period. For a single bank, this value is represented by the sum of the market rates up to the last day of the reserve maintenance period.

\subsubsection{The open market operation at $t=1$}

The initial level of reserves is injected through an open market operation that takes place at the beginning of day 1 , before the market opens. We have assumed that the central bank supplies reserves inelastically at the interest rate $i^{\circ}$ through loans with a maturity of $T=2$ days. Also, all banks participating in the open market operation are identical. They have no funds and have to accumulate $R$ reserves through the next $T=2$ days. The problem for bank $j$ is to choose the reserves borrowed from the central bank, $a_{1}^{j}$, to maximize expected profits for the maturity of the loan, $T=2$ days. Because the OMO is conducted before aggregate uncertainty is resolved, banks take expectations over possible realizations of the aggregate liquidity drain, $\mu$. These expected profits are, therefore,

$$
\pi_{\text {omo }}^{j}=E\left[V_{1}\left(R, a_{1}^{j} ; \mu\right)\right]-2 i^{o} a_{1}^{j} .
$$

The first order condition of (23) determines the initial level of reserves for each bank, $a_{1}^{j}$ as

$$
E\left[\frac{\partial V_{1}\left(R, A_{1} ; \mu\right)}{\partial a_{1}^{j}}\right]=2 i^{o} .
$$

Using (22) and the distribution of aggregate states, implies

$$
i^{o}=\frac{1}{2} E\left[\frac{\partial V_{1}\left(R, A_{1} ; \mu\right)}{\partial a_{1}^{j}}\right]=p^{\mu}\left[\frac{i_{1}(e)+i_{2}(e)}{2}\right]+\left(1-p^{\mu}\right)\left[\frac{i_{1}(0)+i_{2}(0)}{2}\right] .
$$

Notice the bank considers itself a small player in the economy so that its bid in the open market operation does not affect equilibrium rates. The intuition 
from expression (24) should be clear. Banks bid for official reserves up to the point where the daily marginal cost of those reserves (the OMO rate) equals the expected average marginal value of those reserves throughout the maturity of the loan given by the average of the sequence of market rates for each possible realization of the aggregate state weighted by the probability of occurrence of those states. In this sense the OMO rate serves as an attractor of the market rates.

Expression (24) provides an intuition as of how the three official rates, namely, the lending rate, $i^{l}$, the deposit rate, $i^{d}$, and the OMO rate, $i^{o}$, affect the demand for reserves at the open market operation and, therefore, influence the level of excess reserves for the maintenance period. In general, when the OMO rate is closer to the lending rate than to the deposit rate, commercial banks understand that reserves are expensive and bid a smaller amount. With fewer reserves the probability of having an overdraft increases and so does the market rate on average over the period. Furthermore, using (10), (13), (16) and (21) we can see how the demand for reserves at the OMO is independent of the spread between the lending and deposit rates. From (10), (13), and (16) we can write, respectively,

$$
\begin{gathered}
i_{2}(\mu)-i^{d}=\left[p^{f}\left(i^{o}-i^{d}\right)+\left(1-p^{f}\right)\left(i^{l}-i^{d}\right)\right] G\left(r_{2}^{j}+b_{2}^{j}-a_{2}^{j}\right), \\
i_{2}(\mu)-i^{d}=p^{f}\left(i^{o}-\right) i^{d}+\left[\left(i^{l}-i^{d}\right)-p^{f}\left(i^{o}-i^{d}\right)\right] G\left(r_{2}^{j}+b_{2}^{j}-a_{2}^{j}\right), \\
i_{2}(\mu)-i^{d}=\left(i^{l}-i^{d}\right) G\left(R_{2}-A_{2}\right),
\end{gathered}
$$

where both $A_{2}$ and $R_{2}$ depend on $A_{1}$ and are defined in (4) and (5), respectively. On the other hand, from (21) we can write

$$
\begin{aligned}
i_{1}(\mu)-i^{d}= & \left(i^{l}-i^{d}\right) G\left(-A_{1}-\mu\right)+ \\
& +\left(i_{2}(\mu)-i^{d}\right)\left[G\left(R-A_{1}-\mu\right)-G\left(-A_{1}-\mu\right)\right] .
\end{aligned}
$$

Define the asymmetry coefficient of the interest rate corridor as

$$
\alpha=\frac{i^{o}-i^{d}}{i^{l}-i^{d}} .
$$

From (24), (25), (26), (27), and (28) it is easy to see that the demand for funds at the initial OMO, $A_{1}$, that satisfies (24) only depends on $\alpha$ and not on the spread $\left(i^{l}-i^{d}\right)$. Therefore, the central bank can control the bid of banks at the initial OMO by carefully chosen the index of asymmetry $\alpha$. In particular, for given values for width of the interest rate corridor $\left(i^{l}-i^{d}\right)$ and rate of the main refinancing operation, $i^{o}$, the central bank can determine the combination of rates for the standing facilities, $i^{l}$ and $i^{d}$, that ensures its desired liquidity conditions, $A_{1}$, in the overnight market. Of course, this choice for $A_{1}$ is dependent on the rest of parameters of the model, namely, the level of required reserves, $R$, the standard deviation of the liquidity shock, $\sigma$, the subjective probability of a fine-tuning operation, $p^{f}$, the subjective probability of a liquidity crisis, $p^{\mu}$, and the mean of the liquidity shock in the event of a liquidity crisis, $e$. 


\subsection{A numerical approximation}

To get a quantitative assessment as of how the asymmetry of the interest rate corridor affects the demand for excess reserves, we approximate the solution by means of a numerical exercise. In our numerical example we take $i^{o}=3$ percent, $R=200, e=-200, p^{\mu}=0.4$ and $\sigma=80$. Computations are done for different combinations of the lending and deposit rates so we explore the effects of corridors for the overnight rates not centered around the main refinancing rate. We also assume $p^{f}=1$ so that there is a fine-tuning operation for sure. Below we perform computations for different values of this parameter to assess the role of the end-of-period fine-tuning operation.

Imagine the central bank of this economy sets the deposit rate at $i^{d}=2$ percent and the lending rate at $i^{l}=4$ percent. This would imply a symmetric, 200 basis point corridor for the overnight rate, centered around the rate of the main refinancing operation. With these parameter values, market participants would ask for an amount of excess reserves equal to 89 percent of reserve requirements.

\subsubsection{The role of the asymmetric corridor}

To see the role played by the deposit and lending facilities on the determination of the demand for reserves at the main refinancing operation, imagine the central bank keeps the OMO rate constant but shifts the corridor by moving simultaneously the lending and deposit rate up and down. Figure 7 shows the excess reserves demanded at the main refinancing OMO for different values of the asymmetry index of the interest rate corridor defined in (29). Excess reserves are defined as

$$
\xi=\frac{A_{1}-(R / T)}{R / T},
$$

that is, they are computed as the difference between the allotment in the OMO $\left(A_{1}\right)$ and daily reserve requirements $(R / T)$ as a fraction of daily reserve requirements. The figure plots the results for three different values of the probability of the ECB conducting the fine tuning-operation, $p^{f}$. These values are $0,0.5$ and 1 . Because the effect of asymmetry is equivalent for all values of the probability we can concentrate on the continuous line which depicts excess reserves for $p^{f}=1$. The figure shows there is a negative relation between the demand for excess reserves and the asymmetry index. As the OMO rate gets closer to the upper edge of the band (so that the asymmetry index goes to 1), there are less incentives to demand reserves in the refinancing operation both because excess reserves are relatively less remunerated, since the spread between the $\mathrm{OMO}$ rate and the deposit rate is large, and reserve deficiencies are relatively less penalized, since the spread between the lending rate and the OMO rate is small. Also, the figure shows this relation is highly non lineal.

Figures 7 indicates that, in the event of a sure fine-tuning operation at the end of the reserve maintenance period, the central bank could eliminate the autonomous demand for excess reserves leaving interest rates the same range for fluctuation by maintaining the same spread between the lending and deposit 
rates. For that, the central bank would need to set an asymmetric band around the OMO rate. As the figure suggests, given the OMO rate of $i^{\circ}=3$ percent, the central bank could have reduced the excess reserves to zero using an interest rate corridor with index of asymmetry equal to 0.935 . For a 200 basis points width for the corridor the deposit rate should have been $i^{d}=1.13$ percent and a lending rate of $i^{l}=3.13$.

\subsubsection{The role of the fine-tuning operation}

Figure 7 shows excess reserves for different probabilities of the central bank conducting the fine-tuning OMO. These probabilities are $p^{f}=0, p^{f}=0.5$ and $p^{f}=1$. Given the prospects of a large aggregate liquidity drain, banks care about the risk associated with using the lending facility on either of the two days of the maintenance period. As discussed above, banks will find it more profitable to ensure against the risk of going to the lending facility by bidding larger amounts at the initial OMO the closer the rate of the main refinancing operation is to the deposit rate. What Figure 7 shows is that the rate at which they want to use this insurance depends on the existence of the fine-tuning operation at the end of the maintenance period.

First, we start with the case of $p^{f}=0$. This means that there will not be a fine-tuning operation at the end of the reserve maintenance period so that the only source of funds from the central bank, apart from the standing facilities, is the OMO performed at the beginning of day $t=1$. This particular demand function presents a very elastic portion at $\alpha=p^{\mu}=0.4$. Apart from that, the demand is very inelastic around excess reserves $\xi=2$ and $\xi=0$. To understand the shape of this demand function, we can use expression (24) normalized by subtracting the deposit rate $i^{d}$ and dividing by width of the interest rate corridor $\left(i^{l}-i^{d}\right)$ so that all rates are expressed as the asymmetry index

$$
\alpha=\frac{p^{\mu}}{2}\left[\frac{i_{1}(e)-i^{d}}{i^{l}-i^{d}}+\frac{i_{2}(e)-i^{d}}{i^{l}-i^{d}}\right]+\left(\frac{1-p^{\mu}}{2}\right)\left[\frac{i_{1}(0)-i^{d}}{i^{l}-i^{d}}+\frac{i_{2}(0)-i^{d}}{i^{l}-i^{d}}\right] .
$$

As argued above, (30) together with expressions (25), (26), (27), and (28) determine the demand for funds at the initial OMO, $A_{1}$. Thus, banks bid for reserves at the main refinancing OMO to equate the (normalized) rate of the main refinancing operation, as defined by the asymmetry index $\alpha$, with the (normalized) expected value of those reserves during the whole maintenance period, as captured by the right-hand side of (30).

When $p^{f}=0$, the expected value of funds is basically constant and equal to $p^{\mu}$ for all demands for funds at the initial OMO that imply an excess reserve index between $\xi=2$ and $\xi=0$. To see this, remember that with probability $p^{\mu}$ banks expect an aggregate liquidity drain as percentage of reserve requirements of $e /(R / T)=-2<0$. Therefore, for all demands of funds that do not fully compensate for that reserve shortage of $e /(R / T)$, that is, demands which imply excess reserves indices between $\xi=2$ and $\xi=0$ two events may happen. On the one hand, if the aggregate liquidity drain occurs (with probability $p^{\mu}$ ), there will be an aggregate liquidity shortage and rates will be close to the lending rate 
on both days of the maintenance period, so that $i_{1}(e) \simeq i_{2}(e) \simeq i^{l}$ on expression (30). On the other hand, if the aggregate liquidity drain does not occur (with probability $1-p^{\mu}$ ), there will be an aggregate excess of reserves and rates will be close to the deposit rate on both days of the maintenance period, so that $i_{1}(0) \simeq i_{2}(0) \simeq i^{d}$ on expression (30). Therefore, for all those demands for reserves the expected marginal value of funds as measured by the right-hand side of (30) would be

$$
\frac{p^{\mu}}{2}\left[\frac{i^{l}-i^{d}}{i^{l}-i^{d}}+\frac{i^{l}-i^{d}}{i^{l}-i^{d}}\right]+\left(\frac{1-p^{\mu}}{2}\right)\left[\frac{i^{d}-i^{d}}{i^{l}-i^{d}}+\frac{i^{d}-i^{d}}{i^{l}-i^{d}}\right]=p^{\mu} .
$$

Notice that, because the size of the liquidity drain, $e$, is large relative to the standard deviation of the liquidity shock, $\sigma$, the marginal value of funds will be basically constant at $p^{\mu}$ for bids that do not fully compensate the liquidity drain $\mu=e$. In other words, demands that imply an excess reserve index between $\xi=2$ and $\xi=0$ are relatively elastic at the asymmetry index $\alpha=p^{\mu}$. This is the vertical segment corresponding to the line representing the excess reserves for $p^{f}=0$. Thus, if the asymmetry index is below $p^{\mu}$, funds are cheap and banks are willing to compensate for most of the possible liquidity drain (up to the variability in funds associated with the distribution of individual shocks, $\sigma$ ). On the contrary, if the asymmetry index is above $p^{\mu}$ funds are expensive and banks do not have incentives to demand excess reserves.

In the opposite case, when the fine-tuning operation occurs with certainty so that $p^{f}=1$, the reasoning is different. In that case, the fine-tuning operation provides a second chance for banks to obtain liquidity deficiencies or to place excess reserves. Furthermore, the central bank will absorb or provide any liquidity needed at the same rate of the main refinancing operation, $i^{\circ}$. This makes the expected marginal value of funds on any day to be approximately equal to the rate of the main refinancing operation so that the right-hand side of (30) would be

$$
\frac{p^{\mu}}{2}\left[\frac{i^{o}-i^{d}}{i^{l}-i^{d}}+\frac{i^{o}-i^{d}}{i^{l}-i^{d}}\right]+\left(\frac{1-p^{\mu}}{2}\right)\left[\frac{i^{o}-i^{d}}{i^{l}-i^{d}}+\frac{i^{o}-i^{d}}{i^{l}-i^{d}}\right]=\alpha .
$$

independently on whether the aggregate liquidity drain is realized or not. Thus, in the model the only consideration for banks is to insure against the risk of going to the lending facility on the first day of the maintenance period because of a current account overdraft. Therefore, the demand is relatively inelastic at that level. The demand for funds for probabilities of the fine-tuning operation between 0 and 1 , are just in between of the two extreme cases.

Figure 8 shows market interest rates on each day of the maintenance period for a symmetric corridor as a function of the probability that the central bank conducts the fine-tuning OMO. These computations assume that, ex-post, there is no aggregate liquidity drainage. To make the figure comparable with the other figures above, we have computed the relative position of market rates within the interest rate corridor by subtracting the rate of the deposit facility $i^{d}$ and dividing by width of the interest rate corridor $\left(i^{l}-i^{d}\right)$. In Figure 8 
we call this measure the market asymmetry index. The results agree with the data in that, in general, for a symmetric corridor interest rates are depressed for the whole maintenance period as the position of the overnight rate is below the mid point of the interest rate corridor. Of course, as the probability of the fine-tuning operation increases, overnight rates also increase. This is because excess reserves are remunerated at $i^{o}>i^{d}$ whenever the fine-tuning operation occurs.

\section{Conclusions and policy implications}

In periods of financial turmoil, operating frameworks characterized by full allotments and standing facilities, such as the one maintained by the ECB, could provide incentives for banks to demand larger amounts of excess reserves which are then deposited at the deposit facility. We have argued that this is not a desirable situation from the point of view of a central bank. In an environment of financial distress funds do not reach those institutions that need them most and the central bank looses control of liquidity conditions in the interbank market.

We have built a model in which banks have a strong preference for liquidity driven by the prospects of tough liquidity conditions in the future. One possible rationalization of this kind of situation is what Eisenschmidt and Tapking [5] call a funding liquidity risk premium. For these authors, banks internalize the liquidity premium they could have to pay in case they are short of liquidity in the future. This makes them increase their demand for liquidity beyond what could be explained by just counterparty credit risk.

In our model the demand for excess reserves can be interpreted as the coverage bought by banks against the prospective of future tough liquidity conditions. In this sense, banks use the open market operations and the deposit facility as a storage device. How much "coverage" they want to secure, for a given value of the volatility of shocks, will be a function of the price of holding reserves. When we have full allotment at the main refinancing operation the relative prices of this "insurance" can be computed as the index of asymmetry $\alpha$ defined by the relative position of the $\mathrm{OMO}$ in the interest rate corridor. Banks can get reserves at the OMO rate $i^{\circ}$. In the event they are short on liquidity and have to go to the lending facility they will have to pay a premium equal to the spread between the lending and the main refinancing rate, $i^{l}-i^{o}$. If they hold one unit of excess reserves, they will have to pay an opportunity cost equal to the difference between the OMO rate and the deposit rate, $i^{o}-i^{d}$. Notice that the demand for funds is endogenous. Because this demand equates the marginal cost of funds (equal to the OMO rate, $i^{o}$ ) with the marginal expected value of those funds throughout the reserve maintenance period the probabilities of going to the standing facilities are already included in $i^{o}$. Thus, the only way to affect the demand for excess reserves at the initial open market operation is to change the relative price of using the storage device. This can only be done by modifying the index of asymmetry, that is, by moving the relative position of the OMO rate with respect to the rates of the standing facilities which modifies 
the spreads $i^{l}-i^{o}$ and $i^{o}-i^{d}$.

In our model, the width of the interest rate corridor does not have any effect on the demand for reserves as long as the index of asymmetry of the corridor stays constant. Whether this is true or not in the data is still an open empirical question. In any case, the model may be a useful starting point to think of scenarios in which the spread between the rates of the standing facilities does have an effect on the demand for excess reserves.

Finally, we have analyzed how the existence of a fine-tuning operation on the last day of the maintenance period interacts with the demand for funds at the main refinancing operation. Because the fine-tuning OMO fixes the value of funds when central banks evaluate whether reserve requirements are satisfied or not, this OMO affects the determination of the expected marginal cost of reserves throughout the whole maintenance period. Thus, the existence of end of period fine-tuning operations has implications for the optimal bid of banks at the main refinancing operation. We have shown how it decreases the elasticity of demand at the main refinancing OMO.

\section{References}

[1] Ayuso, J. and R. Repullo (2001): "Why did the banks overbid? An empirical model of the fixed rate tenders of the European Central Bank", Journal of International Money and Finance 20 (6), pp. 857-870.

[2] Ayuso, J., and R. Repullo (2003): "A Model of the Open Market Operations of the European Central Bank", The Economic Journal 113 (490), pp. 883-902.

[3] Cassola, N. and C. Morana (2008): "Modelling short-term interest rate spreads in the euro money market", International Journal of Central Banking 4 (4), pp. 1-37.

[4] Demiralp, S. and E. Artuc (2007): "Discount Window Borrowing after 2003: The Explicit Reduction in Implicit Cost" Koc University Working Paper 0708 .

[5] Eisenschmidt, J. and J. Tapking (2009): "Liquidity risk premia in unsecured interbank markets" ECB Working Paper 1025.

[6] Eisenschmidt, J., A. Hirsch and T. Linzert (2009): "Bidding behaviour in the ECB's main refinancing operations during the financial crisis" ECB Working Paper 1052.

[7] European Central Bank (2003): "Changes to the Eurosystem's operational framework for monetary policy" ECB Monthly Bulletin, August.

[8] European Central Bank (2005): "Initial Experience with the Changes to the Eurosystem's operational framework for Monetary Policy implementation", ECB Monthly Bulletin, February. 
[9] European Central Bank (2008): "The Eurosystem's open market operations during the recent period of financial market volatility", ECB Monthly Bulletin, May.

[10] European Central Bank (2008): "The Implementation of Monetary Policy in the Euro Area", ECB Monthly Bulletin, November.

[11] European Central Bank (2009): "Economic and Monetary Developments", ECB Monthly Bulletin, February.

[12] Gaspar, V., G. Pérez Quirós and H. Rodríguez Mendizábal (2008): "Interest Rate Dispersion and Volatility in the Market for Daily Funds", European Economic Review 52 (3), pp. 413-440

[13] Hamilton, J. D. (1996): "The Daily Market for Federal Funds" Journal of Political Economy 104 (1): 26-56.

[14] Goodhart, C. (2009): "Liquidity Management" Conference on Financial Stability and Macroeconomic Policy, Jackson Hole, Wyoming.

[15] Linzert, T. and S. Schmidt (2008): "What explains the spread between the euro overnight rate and the ECB's policy rate?", ECB Working Paper 983.

[16] Pérez Quirós, G. and H. Rodríguez Mendizábal (2006): “The Daily Market for Funds in Europe: What Has Changed with the EMU?", Journal of Money, Credit and Banking 38 (1), pp. 91-118

[17] Valimaki, T. (2008): "Why the effective price for the money exceeds the policy rate in the ECB tenders?" ECB Working Paper 981 
Figure 1. Use of the deposit facility as percentage of required reserves

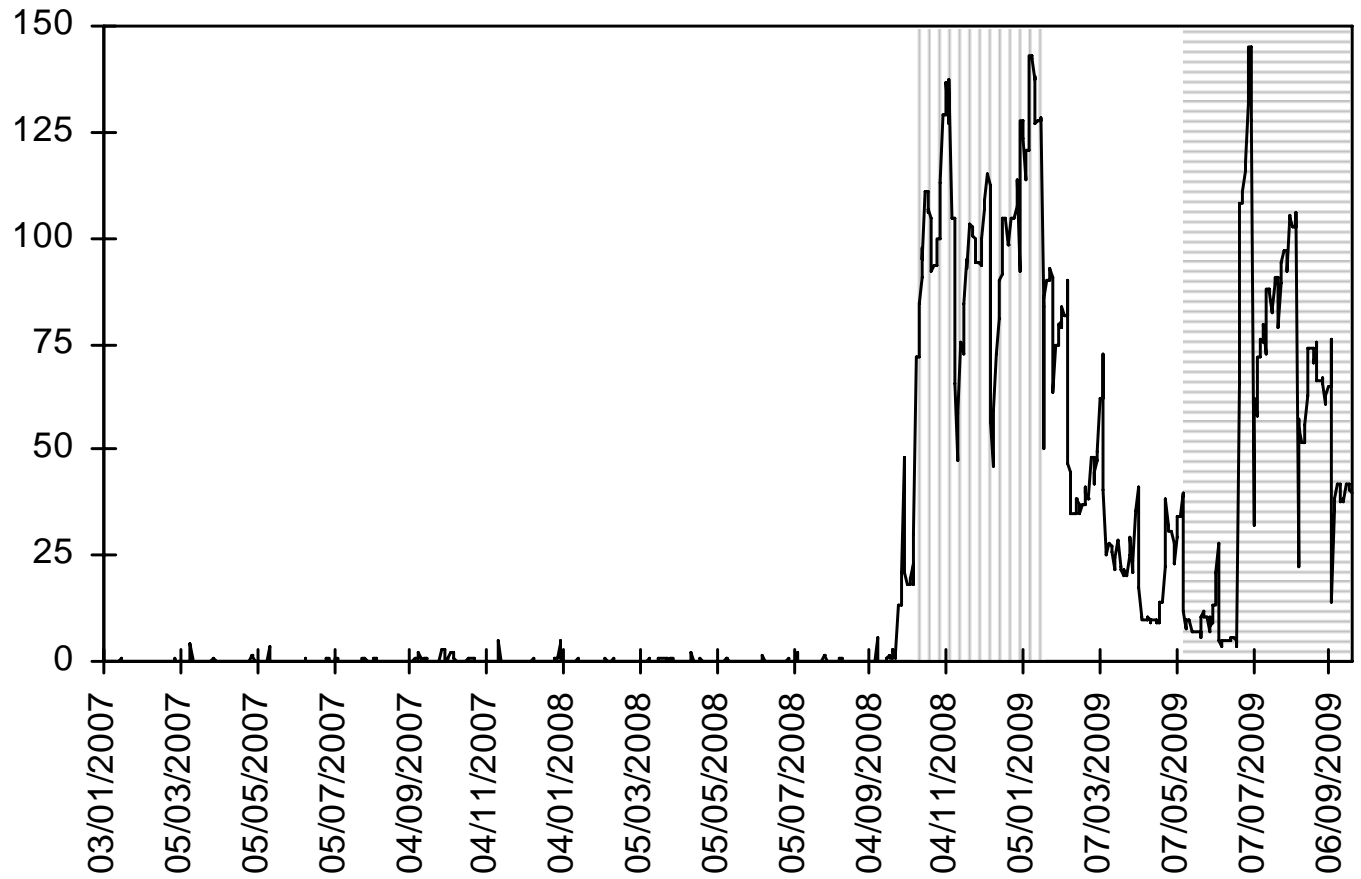

Note: The figure shows the use of the deposit facility as a percentage of required reserves. The sample period covers from January 3rd, 2007 until September 23rd, 2009. The area with vertical lines marks the period when the spread between the lending and deposit rates was 100 basis points. The area with horizontal lines shows the period when the spread between the lending and deposit rate was 150 basis points.

Figure 2. Standing amounts from OMOs and autonomous factors as a percentage

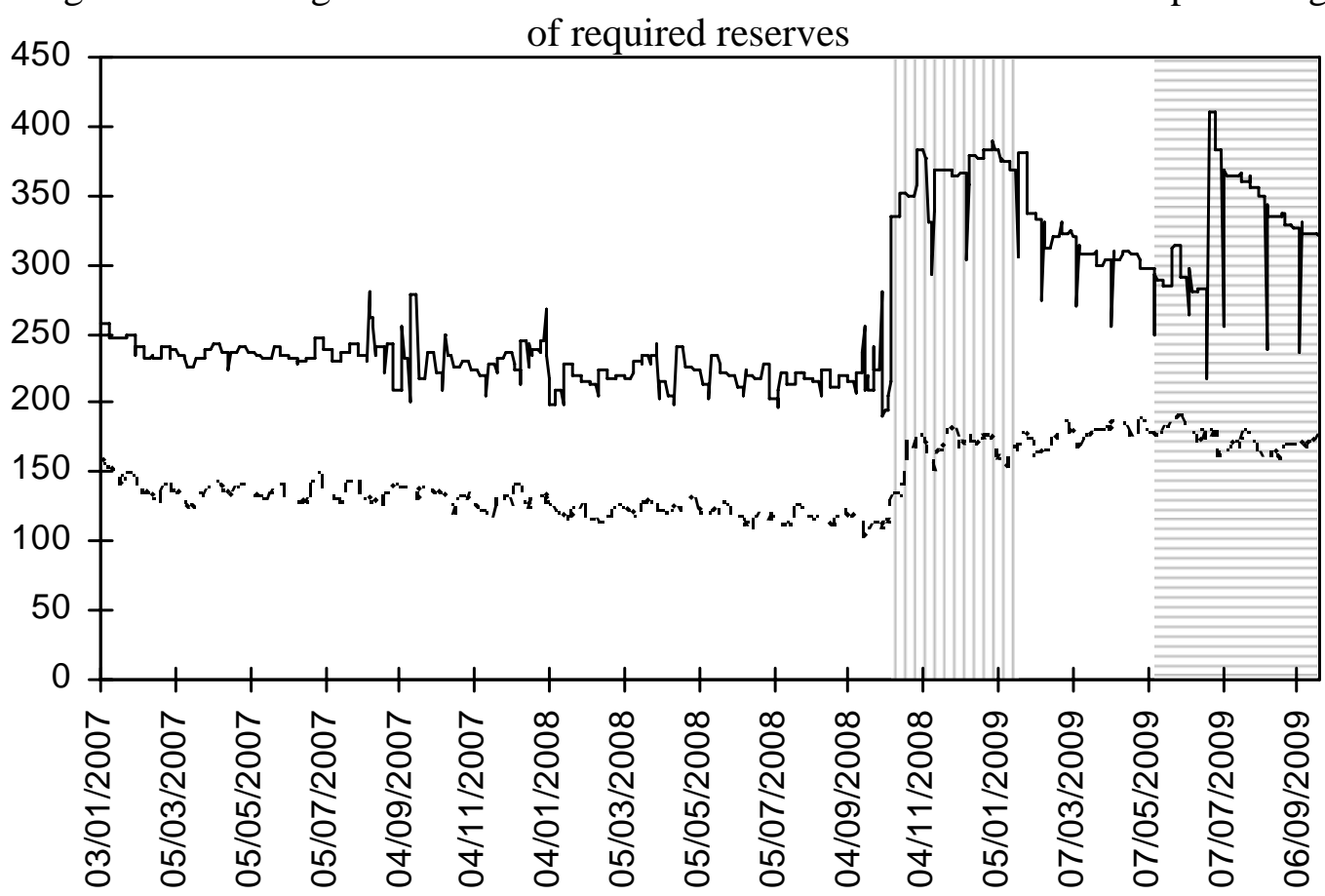

Note: The figure shows the standing amount of reserves allotted at the OMOs (solid) and autonomous factors (dotted) as a percentage of required reserves. The sample period covers from January 3rd, 2007 until September 23rd, 2009. The area with vertical lines marks the period when the spread between the lending and deposit rates was 100 basis points. The area with horizontal lines shows the period when the spread between the lending and deposit rate was 150 basis points. 
Figure 3. Spread between Euribor and Eurepo

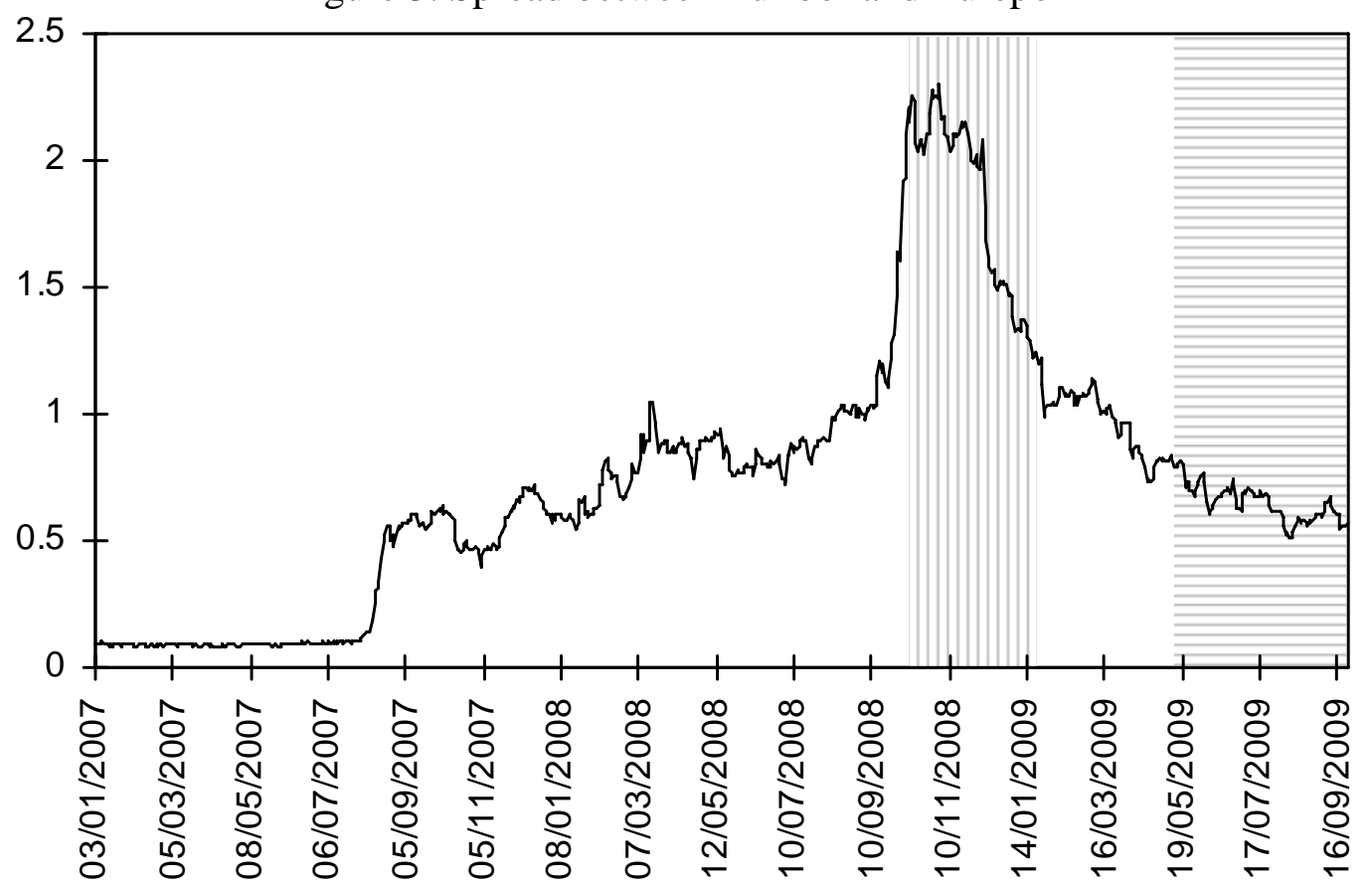

Note: The figure shows the spread between the 12-months Euribor and the 12-months Eurepo. The sample period covers from January 3rd, 2007 until September 23rd, 2009. The area with vertical lines marks the period when the spread between the lending and deposit rates was 100 basis points. The area with horizontal lines shows the period when the spread between the lending and deposit rate was 150 basis points.

Figure 4. Fine-tuning allotment and changes in deposit facility and current account

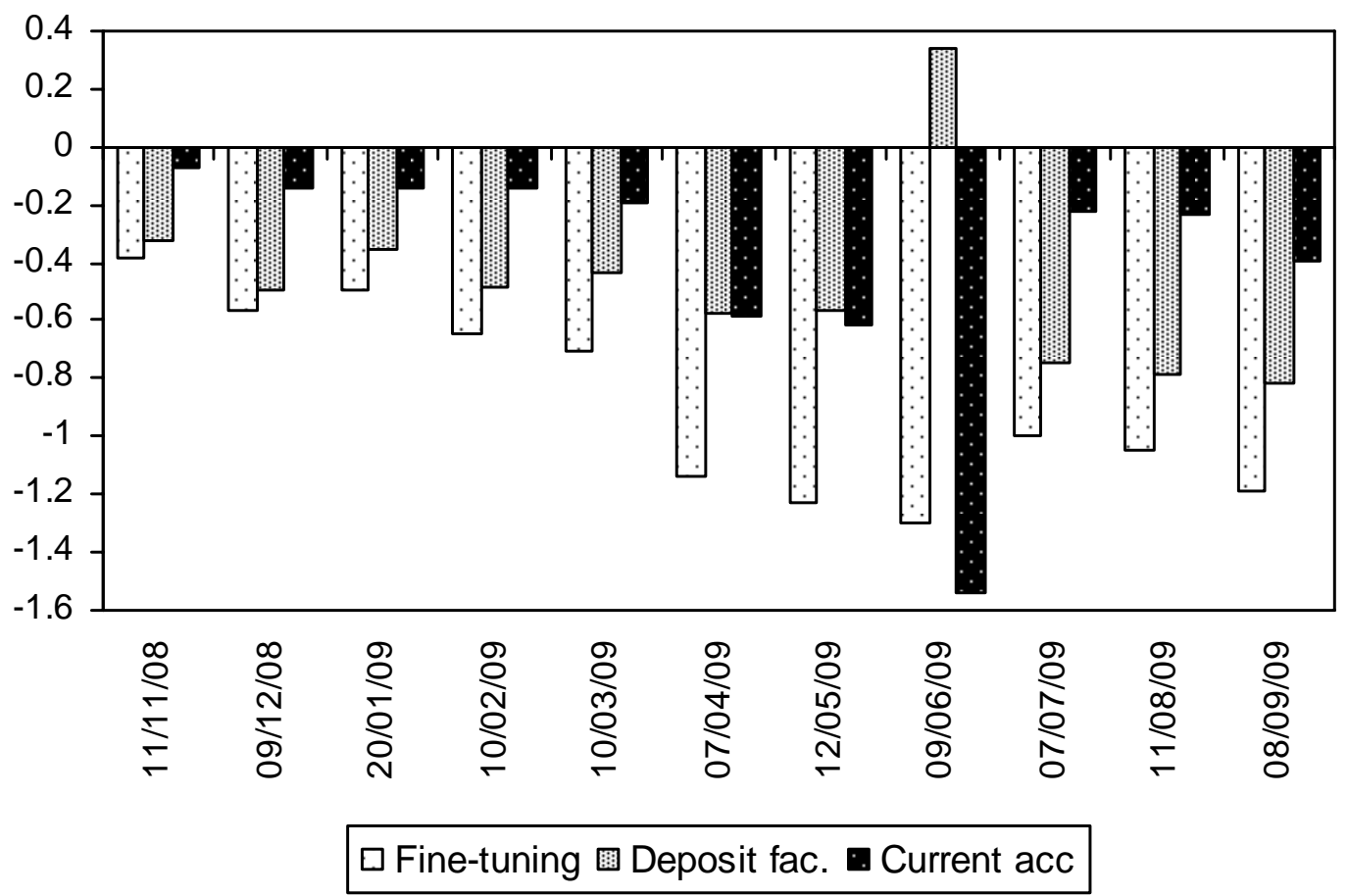

Note: The figure shows the allotment of the fine-tuning operations performed on the last day of the 11 reserve maintenance periods covering the period between November 2008 and September 2009. It also shows the changes in the use of the deposit facility and in the balance of current accounts between the last two days of those reserve maintenance periods. Numbers are as fractions of the use of the deposit facility on the penultimate day of the maintenance periods. 
Figure 5. Spread between Eonia and main refinancing rate

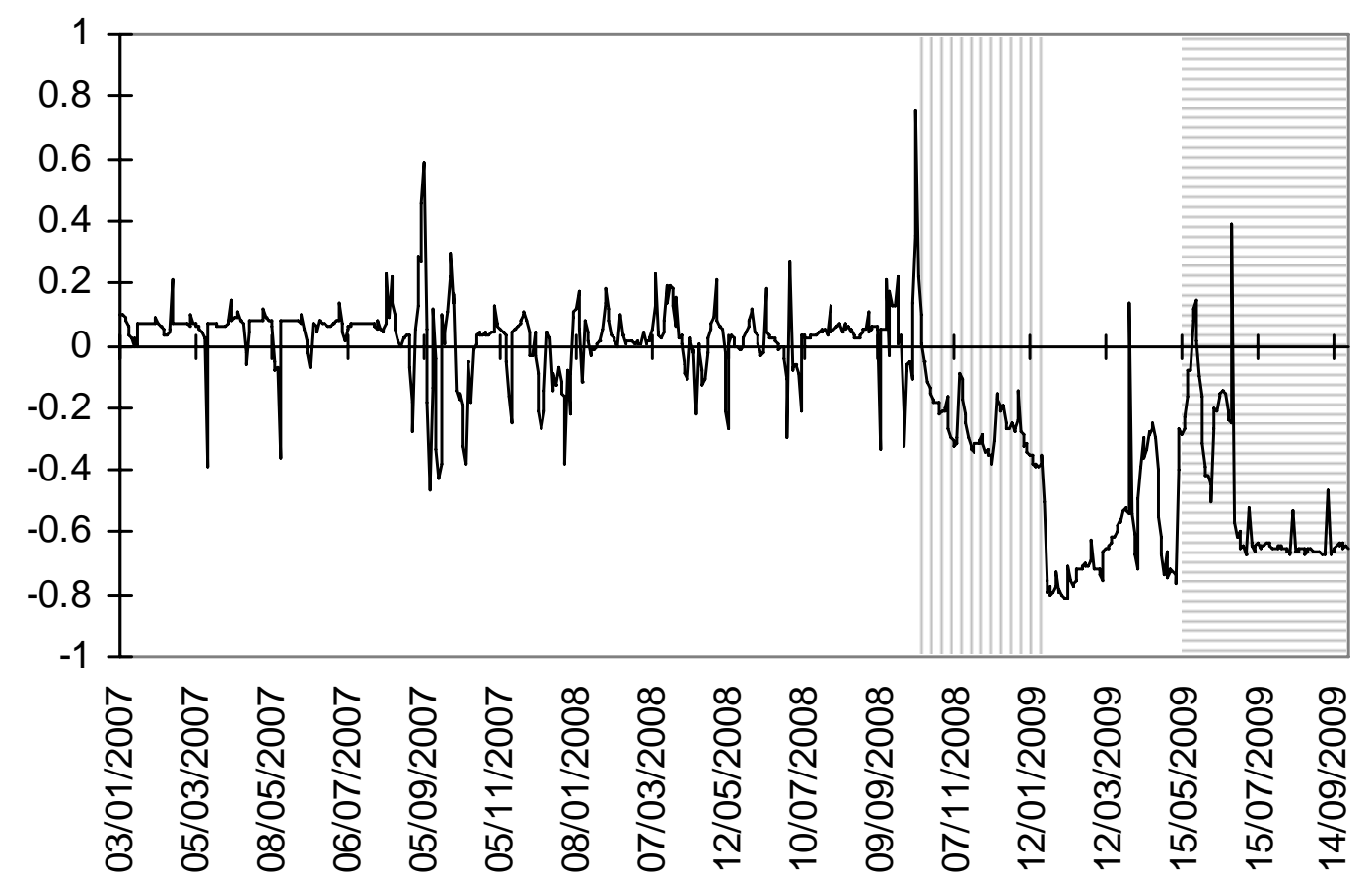

Note: The figure shows the spread between the Eonia and the rate of the main refinancing operations. The sample period covers from January 3rd, 2007 until September 23rd, 2009. The area with vertical lines marks the period when the spread between the lending and deposit rates was 100 basis points. The area with horizontal lines shows the period when the spread between the lending and deposit rate was 150 basis points.

Figure 6. Use of the marginal lending facility as a percentage of required reserves

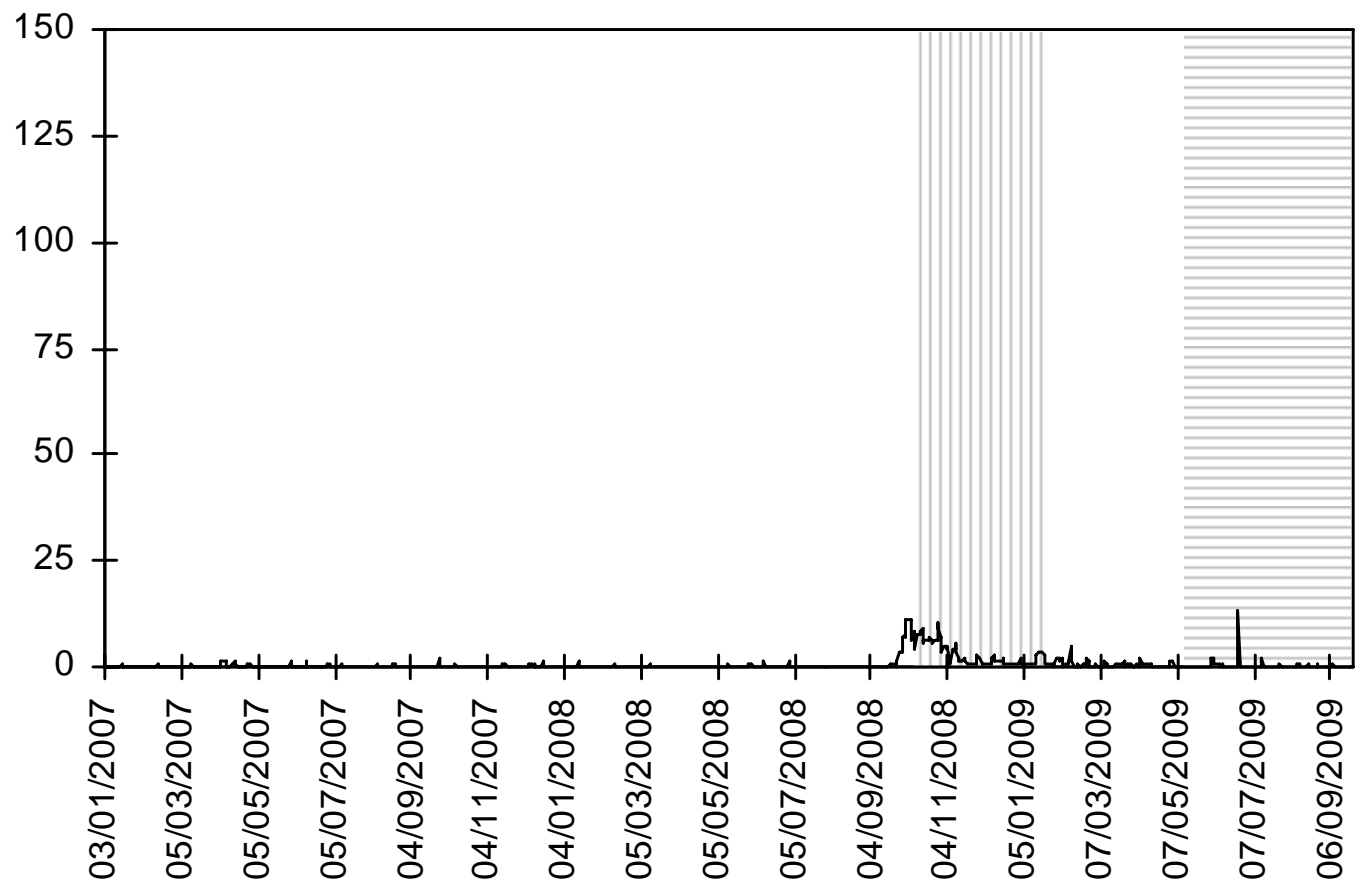

Note: The figure shows the use of the marginal lending facility as a percentage of required reserves. The sample period covers from January 17th, 2007 until July 7th, 2009. The yellow area marks the period when the spread between the lending and deposit rates was 100 basis points. The light blue area shows the period when the spread between the lending and deposit rate was 150 basis points. 
Figure 7. Excess reserves and index of asymmetry

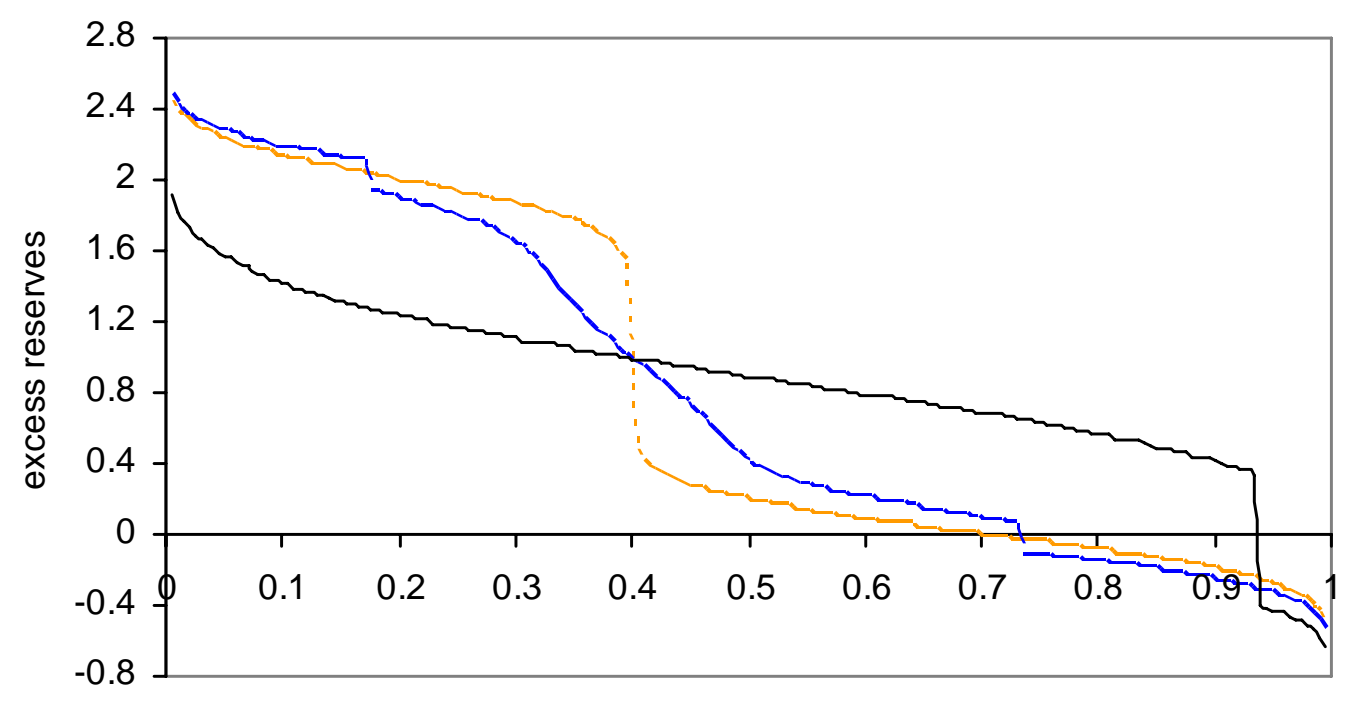

OMO asymmetry index

$$
\cdots \cdots p f=0.0----p f=0.5 \longrightarrow p f=1.0
$$

Note: The black line shows the use of the excess reserves as a fraction of reserve requirements versus the degree of asymmetry of the interest rate corridor measured as the distance between the OMO rate and the deposit rate over the spread between the lending and deposit rate.

Figure 8. Overnight rates for symmetric corridor

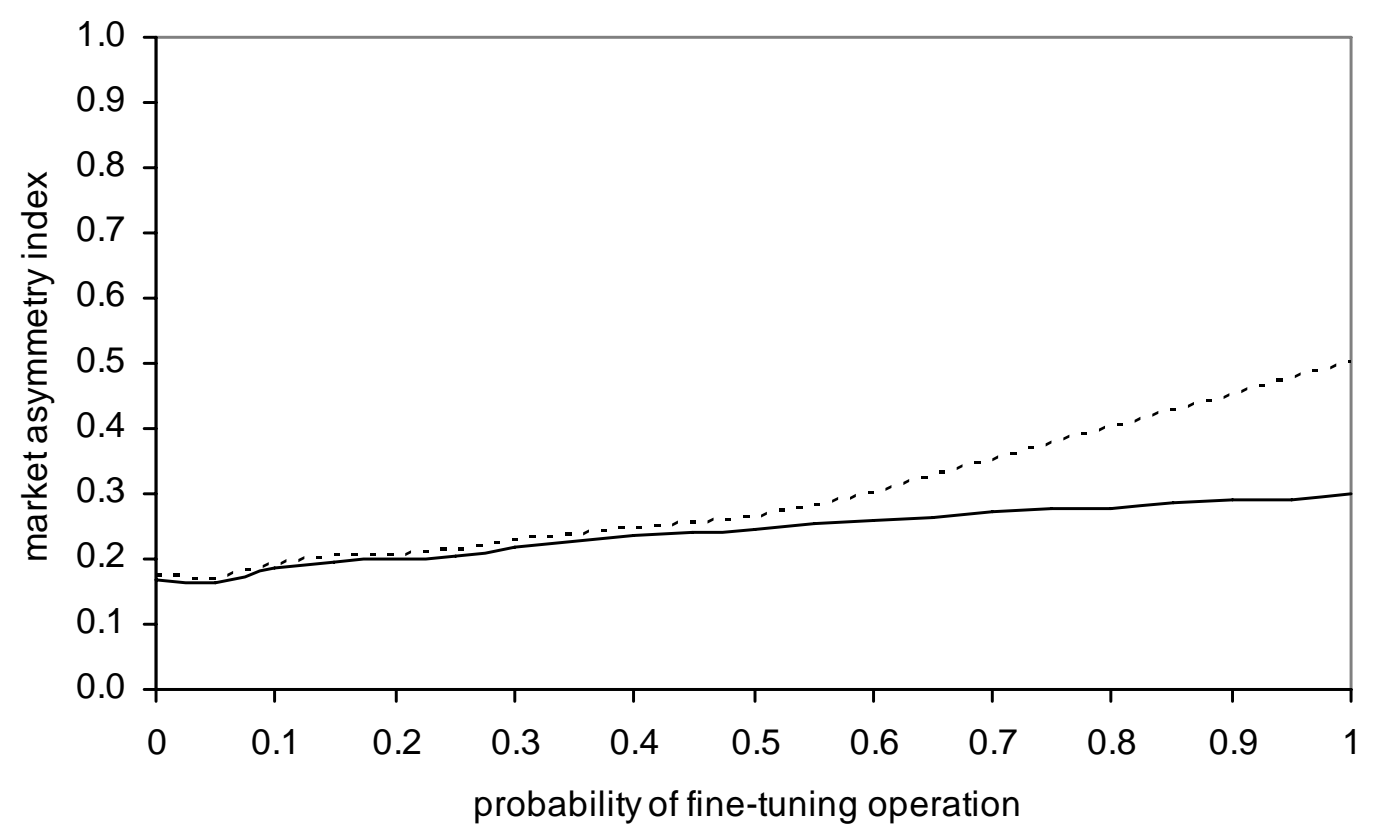

$$
t=1 \cdots \cdot t=2
$$

Note: The lines show the asymmetry index for overnight rates on day 1 (solid) and 2 (dashed) for a symmetric corridor versus the probability of the central bank conducting a fine-tuning operation on the last day of the reserve maintenance period. 